\title{
Calcul des chambres d'équilibre déversantes avec apport de débit
}

\section{Calculations for spilling surge tanks with incoming flow}

\author{
PAR L. ESCANDE
}

MEMBRE DE L'XNSTITUT, INGENIEUR I.E.T.,

DIRECTEUR DE L'ÉCOLE NATIONALE SUPÉRIEURE D'ÉLEGTROTECHNIQUE E'T D'HYDRAULIQUE DE TOULOUSE

I. Calcul approché du volume total déversé sur le seuil d'une cheminée d'équilibre (avec ou sans étranglement) dans le cas où un débit d'apporl tombe directement dans la cheminée, en amont du seuil, au-dessus de l'étranglement lorsqu'il y en $a$ un.

Phase préliminaire al déversement - Première hypothèse pour l'étude du déversement - Etude du déversement - Deuxième hypothèse - Comparaison avec la méthode graphique - Remarque et conclusion.

II. Même calcul dans le cas où le débit d'apport débouche dans la cheminée, au-dessous de l'étranglement éventuel.

Phase préliminaire au déversement - Ftude du déversement dans l'hypothèse d'un seuil de longuear infinie et dans celle d'un seuil de longueur finie - Comparaison avec la méthode graphique et conclusions.
1. Approximation of the volume spilt over the sill of a surge tank, with or without restriction, for the case where the incoming flow falls (a) directly into the tank, (b) upstream of the sill and (c) above the restriction, when there is one. Preliminary phase to spillage - First assumption for spillage investigation - Spillage investigation - Second hypothesis - Comparison with the graphical method - Remarks and conclusion. II. The same calculation for the case where the incoming flow enters the surge tank below a possible restriction.

Preliminary phase to spillage - Spillage investigation based on (a) a sill of infinite length and (b) a sill of finite length - Comparison with the graphical method and conclusions.

\section{CAS OU L'APPORT DE DEBIT S'EFFECTUE A LA PARTIE SUPERIEURE DE L'OUVRAGE}

Dans un travail antérieur (1), nous avons établi les formules et indiqué la méthode permettant de calculer directement le volume total déversé sur le seuil d'une cheminée d'équilibre, comportant ou non un étranglement, à la suite d'un arrêt complet instantané du débit maximum $Q_{0}$ des turbines.

Dans le présent chapitre, nous envisageons le même problème, dans le cas où un débit d'apport $\mathrm{Q}_{a}$ tombe directement dans la cheminée, en amont du seuil, au-dessus de l'étranglement éventuel (fig. 1).

\section{Phase préliminaire au déversement}

$Z$ et A désignant respectivement la cote du plan d'eau, à l'instant $t$, et celle du seuil du

(1) C.R.A.S., t. 235 , p. 338, 1952 et Bulletin de la Societé Francaise des Mécaniciens, $\mathrm{n}^{\circ} 5$, juin 1952 


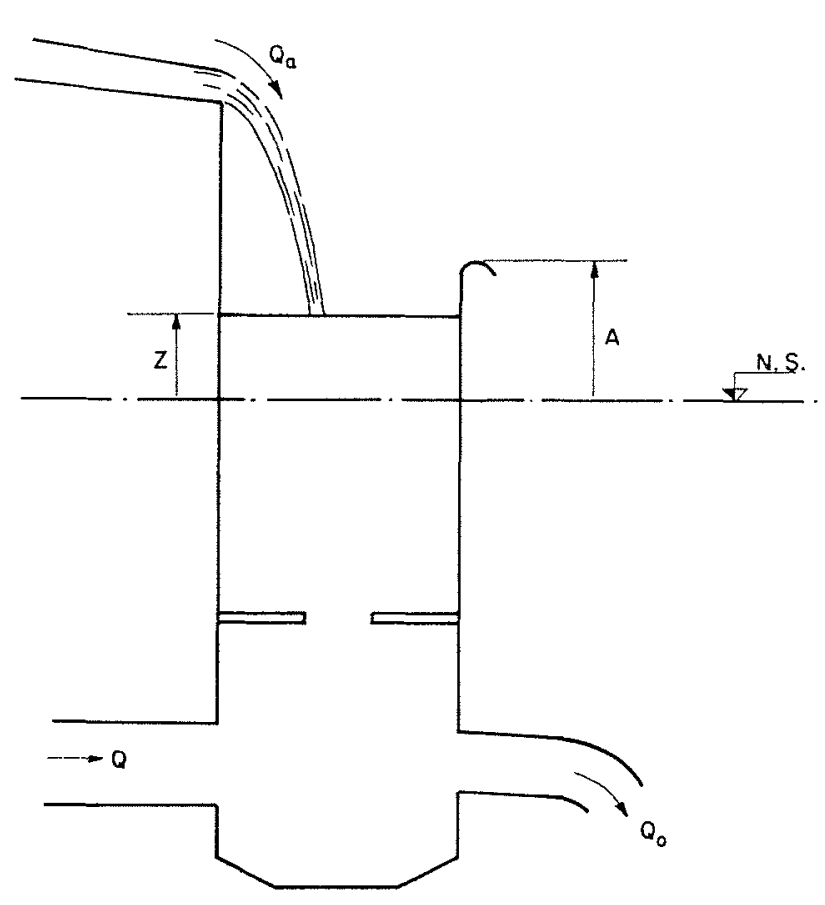

Fli. 1

déversoir, au-dessus du niveau statique, le déversement n'intervient que lorsque $Z$ dépasse $A$.

Jusque-là, l'oscillation intéresse une chambre d'équilibre non déversante, pour laquelle la méthode graphique permet d'étudier la montée, comme nous l'avons montré (1).

Les équations fondamentales des oscillations s'écrivent, dans ce cas, entre grandeurs relatives :

$$
\begin{aligned}
& v(d v / d z)+z+\varepsilon p+\varepsilon^{\prime} r=0 \\
& w=v-w_{a} \\
& p=p_{0} w^{2}=p_{0}\left(v-w_{a}\right)^{2} \\
& r=r_{0} w^{2}=r_{0}\left(v-w_{a}\right)^{2} \\
& \varepsilon=\varepsilon^{\prime} \text { ayant pour valeur absolue l'unité et } \\
& \quad \text { pour signe celui de } w=v-w_{a},
\end{aligned}
$$

avec les notations suivantes :

$\mathrm{L}, f$ longueur et section du canal F section horizontale de la chambre d'équilibre.

W vitesse dans le canal d'amenée, comptée positivement quand l'eau se dirige vers la chambre.

(1) C.R.A.S., t. 224, pp. 185, 537, $895 ; 1947$.

La Houille Blanche, juillet-août et septembre-octobre 1947.
$V \quad$ vitesse ascensionnelle du plan d'eau dans la cheminée d'équilibre.

$W_{0}, V_{0}, U_{0}$ valeurs de $W, V$ el $U$ définies par les relations:

$$
\mathrm{Q}_{0}=f \mathrm{~W}_{0}=\mathrm{FV}_{0}=\mathrm{FU}_{0}
$$

$\mathrm{T}=2 \pi \sqrt{\mathrm{LF} / g f}$ période des oscillations en l'absence de déversement, de pertes de charge, d'étranglement, et de débit d'apport.

$Z_{*}=W_{0} \sqrt{\mathrm{Lf} / g \mathrm{~F}}$ amplitude des oscillations dans les mêmes hypothèses, pour le débit $Q_{0}$.

$P$ el $\mathrm{P}_{0}$ pertes de charge dans le canal d'amenée pour les vitesses respectives $\tilde{W}$ et $W_{0}$.

$R$ et $R_{0}$ pertes de charge dans l'étranglement $\mathrm{R}$ à l'instant $t$ et $\mathrm{R}_{0}$ lorsque l'étranglement est traversé par le débit $Q_{0}$.

$W_{a} \quad$ vitesse définie par la relation $\mathrm{Q}_{a}=f \mathrm{~W}_{a}$.

$v=\frac{\mathrm{V}}{\mathrm{V}_{0}} \quad w=\frac{\mathrm{W}}{\mathrm{W}_{0}} \quad w_{u}=\frac{\mathrm{W}_{u}}{\mathrm{~W}_{0}} \quad z=\frac{\mathrm{Z}}{\mathrm{Z}_{*}} \quad a=\frac{\mathrm{A}}{\mathrm{Z}_{*}}$

$p_{0}=\frac{\mathrm{P}_{0}}{\mathrm{Z}_{*}} \quad p=\frac{\mathrm{P}}{\mathrm{Z}_{*}} \quad r_{0}=\frac{\mathrm{R}_{0}}{\mathrm{Z}_{*}} \quad r=\frac{\mathrm{R}}{\mathrm{Z}_{*}} \quad t^{\prime}=\frac{t}{\mathrm{~T}}$

On remarque que, pendant toute la phase préliminaire au déversement, $W$ reste positif, et l'on a $\varepsilon=\varepsilon^{\prime}=+1$.

Les équalions des oscillations ne sont pas intégrables analytiquement, mais la méthode graphique ci-dessus rappelée (1) permet de déterminer la valeur $w_{1}$ de $w$ à l'instant où débute le déversement.

On en déduit les valeurs $W_{1}=w_{1} W_{0}$ et $\mathrm{Q}_{1}=f \mathrm{~W}_{1}=w_{1} \mathrm{Q}_{0}$ de la vitesse $\mathrm{W}_{1}$ dans le canal d'amenée et du débit $Q_{1}$ débouchant de ce canal dans la chambre d'équilibre à l'instant ou commence le déversement.

\section{Première hypothèse faite pour l'étude du déversement}

$Z$ devenant supérieur à $A$, le déversement commence sous une lame d'abord rapidement croissante, puis maximum et enfin décroissante.

Dans notre calcul, nous raisonnons comme si la longueur du seuil déversant était infiniment srande, la charge commandant le déversement restant pratiquement nulle. 
Autrement dit, nous supposons que $Z$ conserve la valeur A pendant toule la durée du déversement, et nous ne tenons pas compte, de ce fait, des accumulations positives ou négatives à l'intérieur de la chambre.

Les erreurs pouvant résulter de celle hypothèse sont forcément dans le sens de la sécurité : nous faisons intervenir, en effet, pendant tout le déversement, pour le freinage de l'eau en mouvement dans le canal d'amenée, une contre-pression inférieure à la contre-pression réelle du fait de la charge au-dessus du scuil déversant.

\section{Etude du déversement}

Avec l'hypothèse précédente, les équations gínérales s'écrivent :

$$
\begin{aligned}
& \frac{\mathrm{L}}{g} \frac{d \mathrm{~W}}{d t}+\mathrm{A}+\varepsilon(\mathrm{P}+\mathrm{R})=0 \\
& \mathrm{P}+\mathrm{R}=\left(\mathrm{P}_{0}+\mathrm{R}_{0}\right) \mathrm{W}^{2} / \mathrm{W}_{0}{ }^{2} \\
& \mathrm{Q}_{d}=f \mathrm{~W}+\mathrm{Q}_{a}
\end{aligned}
$$

$\mathrm{Q}_{d}$ désignant le débit déversant à linstant $t$.

On a donc l'équation :

$$
\frac{\mathrm{L}}{g} \frac{d \mathrm{~W}}{d l}+\mathrm{A}+\varepsilon \lambda \mathrm{W}^{2}=0
$$

avec :

$$
\lambda=\frac{\mathrm{P}_{0}+\mathrm{R}_{0}}{\mathrm{~W}_{0}^{2}}
$$

dont l'intégration donne, en prenant comme nouvelle origine du temps le début du déversement, tant que Wr est positif, c'est-à-dire tant que $\varepsilon=1$ :

$t=\frac{\mathrm{L}}{g \sqrt{\mathrm{A} \lambda}}\left|\operatorname{arctg} \mathrm{W}_{1} \sqrt{\lambda / \mathrm{A}}-\operatorname{arctg} \mathrm{W} \sqrt{\lambda / \mathrm{A}}\right|$

On en déduit :

$\operatorname{arctg} W \sqrt{\frac{\lambda}{A}}=\operatorname{arctg} W_{1} \sqrt{\frac{\lambda}{A}}-\frac{g \sqrt{A \lambda}}{L} t$

et le débit déversant $\mathrm{Q}_{d l}^{\prime}$ :

$Q^{\prime}{ }_{l}=/ W+Q_{a}=f \sqrt{\frac{A}{\lambda}} \frac{W_{1} \sqrt{\lambda / A}-\lg (g \sqrt{\mathrm{A} \lambda} t / L)}{1+W_{1} \sqrt{\lambda / A} \cdot \lg (g \sqrt{\mathrm{A} \lambda} t / L)}$

Ce dćbit est évidemmenl maximum et égal à :

$$
\mathrm{Q}_{\mathrm{M}}=f \mathrm{~W}_{1}+\mathrm{Q}_{a}=w_{1} \mathrm{Q}_{0}+\mathrm{Q}_{a}
$$

à l'instant zéro du début du déversement, d'après l'hypothèse de base.
Les relations (1), (2), (3) sont valables jusqu'à l'instant $0^{\prime}$ où $W$ s'annule, instant donné, d'après (1), par :

$$
0^{\prime}=\frac{\mathrm{L}}{g \sqrt{\mathrm{A} /}} \operatorname{arctg} \mathrm{W}_{1} \sqrt{\lambda / \mathrm{A}}
$$

Le volume déversé, de 0 à $0^{\prime}$, est donné par :

$$
\Omega^{\prime}{ }_{d}=\int_{0}^{\theta^{\prime}} Q_{d}^{\prime} d t
$$

L'intégration, compte tenu de la valcur (3) de $Q^{\prime}$, s'elfectue en posant :

$$
u=\lg [(g \sqrt{\mathrm{A} \lambda} / \mathrm{I}) t]
$$

ce qui donne :

$$
d t=\frac{\mathrm{L}}{g \sqrt{\mathrm{A} \mathrm{\lambda}^{-}}} \frac{d u}{1+u^{2}} \quad u^{\prime}=\lg \left(\frac{g \sqrt{\mathrm{A} \Lambda}}{\mathrm{L}} 0^{\prime}\right)
$$

el:

$$
Q_{d}^{\prime}=\frac{\mathrm{L}}{g \lambda} \int_{0}^{\prime \prime} \frac{\mathrm{W}_{1} \sqrt{\lambda / \mathrm{A}}-u}{1+\mathrm{W}_{1} \sqrt{\lambda / \mathrm{A}} \cdot u} \cdot \frac{d u}{1+u^{2}}+Q_{t t^{\prime}}
$$

L'intégration de celte fraction rationnelle et la substitution à $\sigma^{\prime}$ de sa valeur (5) donnent finalement :

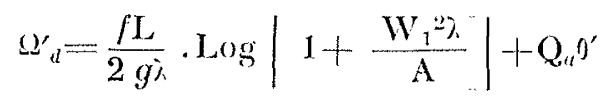

ou :

$$
\begin{aligned}
Q_{d}^{\prime}={ }_{2 g \lambda} \mathrm{L} & \log \left[1+\frac{\mathrm{W}_{3}{ }^{2} \lambda}{\mathrm{A}}\right] \\
& +\frac{\mathrm{Q}_{a} \mathrm{~L}}{g \sqrt{\mathrm{A} \lambda}} \operatorname{arctg} \mathrm{W}_{1} \sqrt{\frac{\lambda}{\mathrm{A}}}
\end{aligned}
$$

Après l'instant $0^{\prime}, W$ devient négatif et $\varepsilon=-1$, de telle sorte que l'équation à integrer s'écrit :

$$
\frac{\mathrm{L}}{g} \frac{d \mathrm{~W}}{d t}+\mathrm{A}-2 \mathrm{~W}^{2}=0
$$

L'intégration de celle équation à variables séparces donne, en eomptant $t$ a partir de l'inslant $0^{\prime}$, pris comme nouvelle origine du temps :

$$
l=\cdots \frac{\mathrm{L}}{2 g \sqrt{\mathrm{A} \lambda}} \cdot \log \frac{1+\mathrm{W} \sqrt{\lambda / A}}{1 \ldots \mathrm{W} \sqrt{\lambda / A}}
$$

On en déduil:

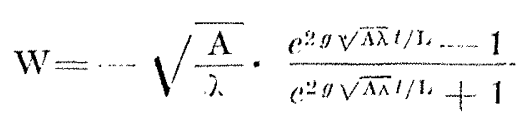

valeur évidemment négalive, el le débit déversant Q. ${ }_{1}^{\prime}$ :

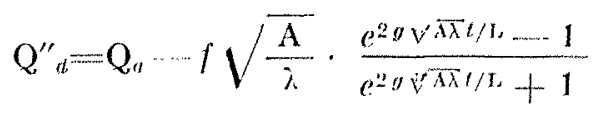


Le facteur qui multiplie $f \sqrt{\mathrm{A} / \lambda}$ étant inférieur à l'unité, pour toutes les valeurs positives de $t$, le débit déversant ne s'annule jamais si l'on $\mathbf{a}$ :

$$
\begin{aligned}
Q_{a}>Q_{r} \text { avec } Q_{r}=f \sqrt{\frac{\mathrm{A}}{\lambda}}=f \mathrm{~W}_{0} & \sqrt{\frac{\mathrm{A}}{\mathrm{P}_{0}+\mathrm{R}_{0}}} \\
= & \mathrm{Q}_{0} \sqrt{\frac{\mathrm{A}}{\mathrm{P}_{0}+\mathrm{R}_{0}}}
\end{aligned}
$$

et il tend asymptotiquement vers la valeur limite $\mathrm{Q}^{\prime \prime}{ }_{d l}$ :

$$
\mathrm{Q}^{\prime \prime}{ }_{a l}=\mathrm{Q}_{a}-\mathrm{Q}_{r}=\mathrm{Q}_{a}-\mathrm{Q}_{0} \sqrt{\mathrm{A} /\left(\mathrm{P}_{0}+\mathrm{R}_{0}\right)}
$$

en un temps infini.

On peut remarquer que :

$$
\mathrm{Q}_{r}=\mathrm{Q}_{0} \sqrt{\frac{\mathrm{A}}{\mathrm{P}_{0}+\mathrm{R}_{0}}}
$$

représente le débit du courant de retour qui, dans le régime permanent final, s'établira asymptotiquement dans le canal d'amenée, débit dont l'expression s'obtient immédiatement en remarquant qu'il s'effectue sous la charge A.

Compte tenu de cette remarque, si l'on a :

$$
\mathrm{Q}_{a}<f^{\prime} \sqrt{\mathrm{A} / \lambda} \text { ou } \mathrm{Q}_{a}<\mathrm{Q}_{r}
$$

le débit déversant s'annule au bout d'un temps fini $\theta^{\prime \prime}$, toujours compté à partir de $\theta^{\prime}$, tel que :

$$
\begin{aligned}
\theta^{\prime \prime}=\frac{\mathrm{L}}{2 g \sqrt{\mathrm{A} \lambda}} & \log \frac{f \sqrt{\mathrm{A} / \lambda}+\mathrm{Q}_{a}}{f \sqrt{\overline{\mathrm{A} / \lambda}}-\mathrm{Q}_{a}} \\
& =\frac{\mathrm{L}}{2 g \sqrt{\mathrm{A} \lambda}} \cdot \log \frac{\mathrm{Q}_{r}+\mathrm{Q}_{a}}{\mathrm{Q}_{r}-\mathrm{Q}_{a}}
\end{aligned}
$$

Dans l'hypothèse (11), le volume déversé, après l'instant $\theta^{\prime}$, est donné par l'expression :

$$
\begin{aligned}
& \Omega^{\prime \prime}{ }_{a}= \int_{0}^{g^{\prime \prime}} \mathrm{Q}^{\prime \prime}{ }_{d} d t=\mathrm{Q}_{a} \theta^{\prime \prime}-f \sqrt{\mathrm{A} / \lambda} \\
& \int_{0}^{\theta^{\prime \prime}} \frac{e^{2 g \sqrt{\mathrm{A} \lambda} t / \mathrm{L}}-1}{e^{2 c \sqrt{\mathrm{A \lambda} t / \mathrm{L}}+1} d t} \\
& \Omega^{\prime \prime}{ }_{d}=\mathrm{Q}_{a} \theta^{\theta^{\prime \prime}}-f \sqrt{\mathrm{A} / \lambda} \int_{0}^{\theta^{\prime \prime}} \operatorname{th}\left(\frac{g \sqrt{\mathrm{A} \lambda}}{\mathrm{L}} t\right) d t \\
& \Omega^{\prime \prime}{ }_{d}=\mathrm{Q}_{a} \theta^{\prime \prime}-f \mathrm{~L} / g \lambda \log \left[\operatorname{ch}\left(\frac{g \sqrt{\mathrm{A} \lambda}}{\mathrm{L}} \theta^{\prime \prime}\right)\right]
\end{aligned}
$$

or :

$$
\frac{g \sqrt{\mathrm{A} \lambda}}{\mathrm{L}} \theta^{\prime \prime}=1 / 2 \log \frac{\mathrm{Q}_{r}+\mathrm{Q}_{a}}{\mathrm{Q}_{r}-\mathrm{Q}_{a}}=\arg \text { th } \frac{\mathrm{Q}_{a}}{\mathrm{Q}_{r}}
$$

et :

$\operatorname{ch}\left(\frac{g \sqrt{\mathrm{A} \lambda}}{\mathrm{L}} \theta^{\prime \prime}\right)=\frac{1}{\sqrt{1-\left(\mathrm{Q}_{a}{ }^{2} / \mathrm{Q}_{r}{ }^{2}\right)}}=\frac{\mathrm{Q}_{r}}{\sqrt{\mathrm{Q}_{r}{ }^{2}-\mathrm{Q}_{a}{ }^{2}}}$ d'où :

$$
\Omega^{\prime \prime}{ }_{d}=\mathrm{Q}_{a}{ }^{\prime \prime} \theta^{\prime \prime}+\frac{f \mathrm{~L}}{2 g \lambda} \log \frac{\mathrm{Q}_{r}{ }^{2}-\mathrm{Q}_{a}^{2}}{\mathrm{Q}_{r}^{2}}
$$

ou :

$$
\begin{aligned}
{\Omega^{\prime \prime}}_{a} & =\frac{\mathrm{Q}_{a} \mathrm{~L}}{2 g \sqrt{\mathrm{A} \lambda}} \log \frac{\mathrm{Q}_{r}+\mathrm{Q}_{a}}{\mathrm{Q}_{r}-\mathrm{Q}_{a}} \\
& +\frac{f \mathrm{~L}}{2 g \lambda} \log \frac{\mathrm{Q}_{r}{ }^{2}-\mathrm{Q}_{a}{ }^{2}}{\mathrm{Q}_{r}{ }^{2}}
\end{aligned}
$$

Le volume déversé total est :

soit :

$$
\Omega_{d}=\Omega^{\prime}{ }_{d}+\Omega^{\prime \prime}{ }_{d}
$$

$$
\begin{array}{r}
\Omega_{d}=\frac{f \mathrm{~L}}{2 g \lambda} \log \left[\left(1+\frac{\mathrm{W}_{1}^{2} \lambda}{\mathrm{A}}\right)\left(\frac{\mathrm{Q}_{r}{ }^{2}-\mathrm{Q}_{a}{ }^{2}}{\mathrm{Q}_{r}{ }^{2}}\right)\right] \\
+\mathrm{Q}_{\mathrm{a}}\left(\theta^{\prime}+\theta^{\prime \prime}\right)
\end{array}
$$

ou encore, en remarquant que :

$$
\begin{gathered}
\mathrm{W}_{1} \sqrt{\frac{\lambda}{\mathrm{A}}}=\frac{\mathrm{W}_{1}}{\mathrm{~W}_{0}} \sqrt{\frac{\mathrm{P}_{0}+\mathrm{R}_{0}}{\mathrm{~A}}}=\frac{f \mathrm{~W}_{1}}{\mathrm{Q}_{r}}=\frac{\mathrm{Q}_{1}}{\mathrm{Q}_{r}} \\
=w_{1} \frac{\mathrm{Q}_{0}}{\mathrm{Q}_{r}}=\frac{\mathrm{Q}_{\mathrm{M}}-\mathrm{Q}_{a}}{\mathrm{Q}_{r}} \\
\Omega_{d}=\frac{f \mathrm{~L}}{2 g \lambda} \log \left[\frac{\left(\mathrm{Q}_{r}{ }^{2}+\mathrm{Q}_{1}{ }^{2}\right)\left(\mathrm{Q}_{r}{ }^{2}-\mathrm{Q}_{a}{ }^{2}\right)}{\mathrm{Q}_{r}{ }^{4}}\right] \\
\quad+\mathrm{Q}_{a}\left(\theta^{\prime}+0^{\prime \prime}\right)
\end{gathered}
$$

avec 0 désignant la durée totale du déversement :

$$
\begin{aligned}
0=\theta^{\prime}+0^{\prime \prime \prime}=\frac{\mathrm{L}}{g \sqrt{\mathrm{A \lambda}}} \\
\quad\left[\operatorname{arctg} \mathrm{W}_{1} \sqrt{\frac{\lambda}{\mathrm{A}}}+1 / 2 \log \left(\frac{\mathrm{Q}_{r}+\mathrm{Q}_{a}}{\mathrm{Q}_{r}-\mathrm{Q}_{a}}\right)\right]
\end{aligned}
$$

ou :

$$
\theta=\frac{\mathrm{L}}{g \sqrt{\mathrm{A} \lambda}}\left[\operatorname{arctg} \frac{\mathrm{Q}_{1}}{\mathrm{Q}_{2}}+1 / 2 \log \left(\frac{\mathrm{Q}_{r}+\mathrm{Q}_{a}}{\mathrm{Q}_{r}-\mathrm{Q}_{a}}\right)\right]
$$

\section{Deuxième hypothèse faite pour l'étude du déversement}

Dans notre travail antérieur concernant les chambres sans débit d'apport, nous avions montré qu'une approximation nettement supérieure pouvait être obtenue, vis-à-vis des résultats auxquels conduisait l'hypothèse du seuil déversant de longueur infinie à la cote $\mathrm{A}$ : il suffisait de remplacer, dans les formules générales relatives à la phase du déversement, obtenues à partir de cette hypothèse, le terme A par le suivant :

$$
\mathrm{A}^{\prime}=\mathrm{A}+0,5 h_{1}
$$

$h_{1}$ désignant la charge nécessaire pour l'écoulement, en régime permanent, au-dessus du déversoir, du débit maximum $Q_{M}$. 
Nous adopterons, comme deuxième hypothèse, le même mode opératoire pour le cas de l'étude actuelle concernant une cheminée avec débit d'apport.

$W_{1}$ et $Q_{M}$ conservant la même valeur que dans la première hypothèse, toutes les formules établies pour celle-ci sont immédiatement applicables en y substituant $A^{\prime}$ à $A$.

\section{Comparaisons avec la méthode graphique classique}

Nous les avons effectuées en considérant plusieurs cas correspondant tous au même canal d'amenée, au même débit total $Q_{0}$ et à une même cheminée d'équilibre déversante :

$$
\left.\begin{array}{l}
\mathrm{L}=4.138 \mathrm{~m} \\
f=3,464 \mathrm{~m}^{2} \\
\mathrm{Q}_{0}=8 \mathrm{~m}^{3} / \mathrm{s} \\
\mathrm{P}_{0}=8,5 \mathrm{~m} \\
\mathrm{~F}=5,55 \mathrm{~m}^{2} \\
\mathrm{~A}=3 \mathrm{~m}
\end{array}\right\} \begin{aligned}
& \text { La longueur du déversoir } \\
& \text { est telle qu'un déversement } \\
& \text { de } 8 \mathrm{~m}^{3} / \mathrm{s} \text { correspond à une } \\
& \text { charge de } 0,75 \mathrm{~m} .
\end{aligned}
$$

Nous avons envisagé le cas d'une chambre ordinaire, d'une part, et d'une chambre à étranglement, d'autre part, et nous avons considéré les diverses valeurs suivantes du débit d'apport :

$$
\begin{array}{ll}
\mathrm{Q}_{a}=\left\{\begin{array}{cc}
0 & \mathrm{~m}^{3} / \mathrm{s} \\
1,5 & \mathrm{~m}^{3} / \mathrm{s} \\
3 & \mathrm{~m}^{3} / \mathrm{s} \\
4,5 \mathrm{~m}^{3} / \mathrm{s}
\end{array}\right\} \text { pour } \mathrm{R}_{0}=0 \\
\mathrm{Q}_{a}=\left\{\begin{array}{cc}
0 & \mathrm{~m}^{3} / \mathrm{s} \\
1,5 & \mathrm{~m}^{3} / \mathrm{s} \\
3 & \mathrm{~m}^{3} / \mathrm{s}
\end{array}\right\} \text { pour } \mathrm{R}_{0}=10 \mathrm{~m}
\end{array}
$$

Le débit de $4,5 \mathrm{~m}^{3} / \mathrm{s}$ conduit, en effet, à un déversement permanent $\left(Q_{a}>Q_{r}\right)$ pour la cheminée à étranglement, contrairement à ce qui se passe pour la chambre ordinaire.

Les tableaux I à VII donnent, en fonction de $\mathrm{Q}_{a}$, pour $\mathrm{R}_{0}=0$ (tableaux I à IV) et pour $\mathrm{R}_{0}=10 \mathrm{~m}$ tableaux V à VII), calculées respectivement par

TABLEAU I

\begin{tabular}{|c|c|c|c|c|c|}
\hline & \multirow{3}{*}{$\mid \begin{array}{c}\text { MÉTHoDE } \\
\text { graph. } \\
\begin{array}{c}\text { valeur } \\
\text { obt. }\end{array}\end{array}$} & \multicolumn{4}{|c|}{ MÉTHOdE ANALYTIQUE } \\
\hline & & \multicolumn{2}{|c|}{ Hypothèse A } & \multicolumn{2}{|c|}{ Hypothèse $A^{\prime}$} \\
\hline & & \begin{tabular}{|l} 
valeur \\
obt.
\end{tabular} & $\left|\begin{array}{l}\text { erreur } \\
\text { en } \%\end{array}\right|$ & $\left|\begin{array}{c|}\text { valeur } \\
\text { obt. }\end{array}\right|$ & $\mid \begin{array}{l}\text { erreur } \\
\text { en } \%\end{array}$ \\
\hline $\mathrm{Q}_{\mathrm{M}} \mathrm{m}^{3} / \mathrm{s}$ & $7,83 \mid$ & 7,88 & 0,638 & 7,88 & 0,638 \\
\hline \multicolumn{2}{|c|}{\begin{tabular}{l|l}
$\theta^{\prime} \mathrm{s} \ldots \ldots$ & 135
\end{tabular}} & 154 & $|14,08|$ & 140 & 3,7 \\
\hline \multicolumn{2}{|c|}{$\theta^{\prime \prime} \mathrm{s} \ldots \ldots .135$} & 143,8 & $|6,52|$ & 125 & $\mid-7,41$ \\
\hline \multicolumn{2}{|c|}{$\theta \mathrm{s} \ldots \ldots .270$} & 297,8 & 10,3 & 265 & $-1,85$ \\
\hline \multicolumn{2}{|c|}{$\Omega_{d} \mathrm{~m}^{3} \ldots \mid 880$} & 988,7 & $|12,35|$ & 897,8 & 2,02 \\
\hline
\end{tabular}

$$
\mathrm{Q}_{a}=0 \mathrm{~m}^{3} / \mathrm{s} \quad \mathrm{R}_{0}=0 \mathrm{~m}
$$

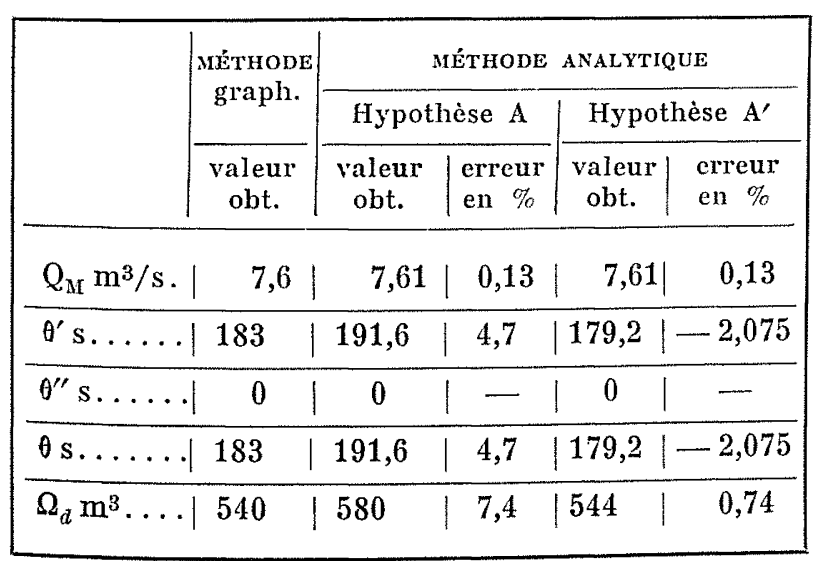

\begin{tabular}{|c|c|c|c|c|c|}
\hline & \multirow{3}{*}{$\left|\begin{array}{c}\text { Ḿтноре } \\
\text { graph. } \\
\begin{array}{c}\text { valeur } \\
\text { obt. }\end{array}\end{array}\right|$} & \multicolumn{4}{|c|}{ MÉTHODE ANALYTIQUE } \\
\hline & & \multicolumn{2}{|c|}{ Hypothèse A } & \multicolumn{2}{|c|}{ Hypothèse $A^{\prime}$} \\
\hline & & $\begin{array}{c}\text { valeur. } \\
\text { obt. }\end{array}$ & $\left|\begin{array}{l}\text { erreur } \\
\text { en } \%\end{array}\right|$ & $\left|\begin{array}{c}\text { valeur } \\
\text { obt. }\end{array}\right|$ & $\mid \begin{array}{l}\text { erreur } \\
\text { en } \%\end{array}$ \\
\hline$Q_{\mathrm{MI}} \mathrm{m}^{3} / \mathrm{s}$ & 7,9 & 7,927 & 0,342 & $|7,927|$ & 0,342 \\
\hline$\theta^{\prime} \mathrm{s} \ldots \ldots$ & $102 \mid$ & 120,3 & $|17,95|$ & $|108,8|$ & 6,66 \\
\hline$\theta^{\prime \prime} \mathrm{s} \ldots \ldots$ & 1305 & 349 & 14,4 & 262 & $-14,1$ \\
\hline$\theta \mathrm{s} \ldots \ldots$ & $407 \mid$ & 469,3 & 15,3 & $|370,8|$ & $-8,9$ \\
\hline$\Omega_{d} \mathrm{~m}^{3} \ldots$ & 1076 & 1255 & 16,6 & $|1110|$ & 2,23 \\
\hline
\end{tabular}

TABLEAU II

$$
Q_{a}=1,5 \mathrm{~m}^{3} / \mathrm{s} \quad \mathrm{R}_{0}=0 \mathrm{~m}
$$

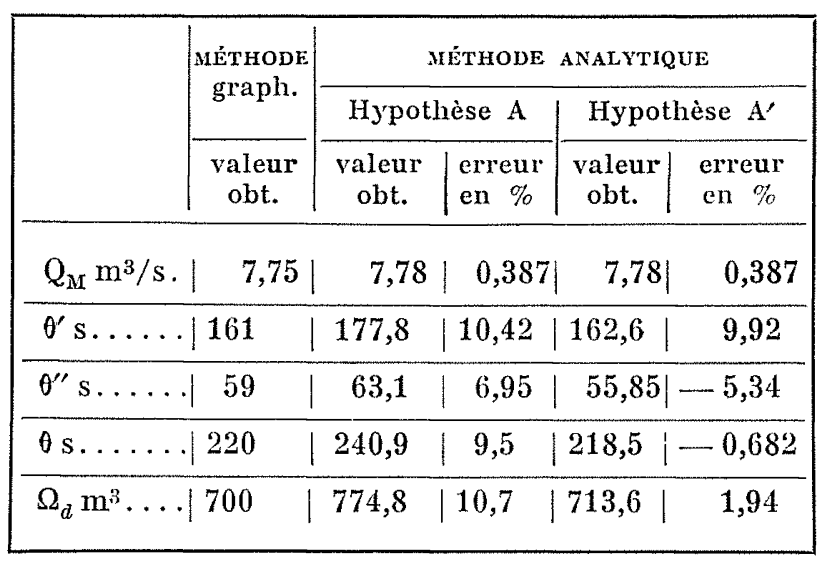

\section{TABLEAU III}

$$
\mathrm{Q}_{a}=3 \mathrm{~m}^{3} / \mathrm{s} \quad \mathrm{R}_{0}=0 \mathrm{~m}
$$

TABLEAU IV

$$
\mathrm{Q}_{a}=4,5 \mathrm{~m}^{3} / \mathrm{s} \quad \mathrm{R}_{0}=0 \mathrm{~m}
$$




\section{TABLEAU V}

$Q_{n}=0 \mathrm{~m}^{3} / \mathrm{s} \quad R_{0}=10 \mathrm{~m}$

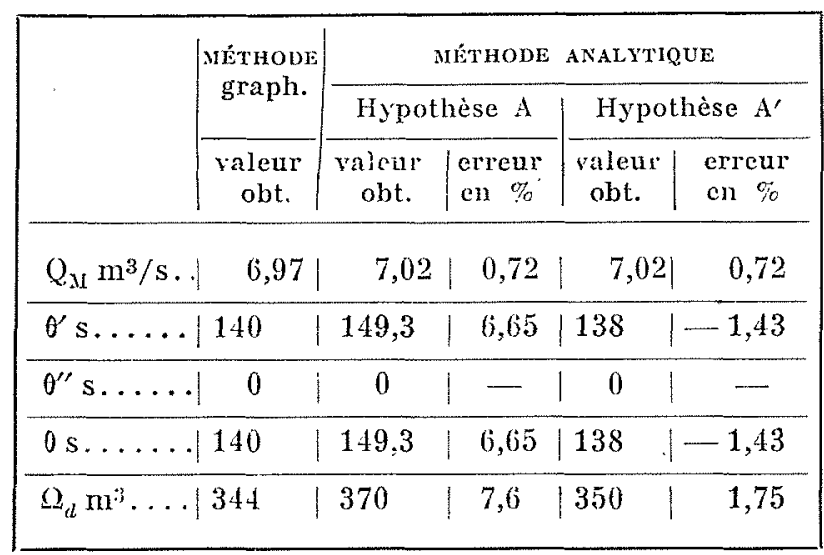

TABLEAU VI

$Q_{a}=1,5 \mathrm{~m}^{3} / \mathrm{s} \quad \mathrm{R}_{0}=10 \mathrm{~m}$

\begin{tabular}{|c|c|c|c|c|c|}
\hline \multirow{3}{*}{ 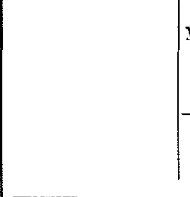 } & \multirow{3}{*}{$\begin{array}{c}\text { MÉthome } \\
\text { graph. } \\
\begin{array}{c}\text { valeur } \\
\text { obt. }\end{array}\end{array}$} & \multicolumn{4}{|c|}{ MÉTHODE ANALYTLQUE } \\
\hline & & \multicolumn{2}{|c|}{ Hypothèse A } & \multicolumn{2}{|c|}{ Hypothèse $A^{\prime}$} \\
\hline & & $\begin{array}{c}\text { valcur } \\
\text { obt. }\end{array}$ & $\begin{array}{l}\text { erreur } \\
\text { en } \%\end{array}$ & $\begin{array}{l}\text { valeur } \\
\text { obt. }\end{array}$ & $\begin{array}{l}\text { erreur } \\
\text { en } \%\end{array}$ \\
\hline $\mathrm{Q}_{\mathrm{MI}} \mathrm{m}^{3} / \mathrm{s}$. & 7,47 & 7,49 & 0,268 & 7,49 & 0,268 \\
\hline \multicolumn{2}{|c|}{$\theta^{\prime} s \ldots \ldots \mid 130$} & 140,7 & 8,24 & $|129,8|$ & $-0,144$ \\
\hline \multicolumn{2}{|c|}{$\theta^{\prime \prime} \mathrm{s} \ldots \ldots \mid 63$} & 66 & 4,76 & $|58,06|$ & $-7,84$ \\
\hline \multicolumn{2}{|c|}{$\theta \mathrm{s} \ldots \ldots \mid 193$} & 206,7 & 7,1 & 187,86 & $-2,66$ \\
\hline \multicolumn{2}{|c|}{$\Omega_{a} \mathrm{~m}^{3} \ldots \mid 528$} & 573 & 8,52 & 531,8 & 0,72 \\
\hline
\end{tabular}

TABLEAU VII

$\mathrm{Q}_{i}=3 \mathrm{~m}^{3} / \mathrm{s} \quad \mathrm{R}_{0}=10 \mathrm{~m}$

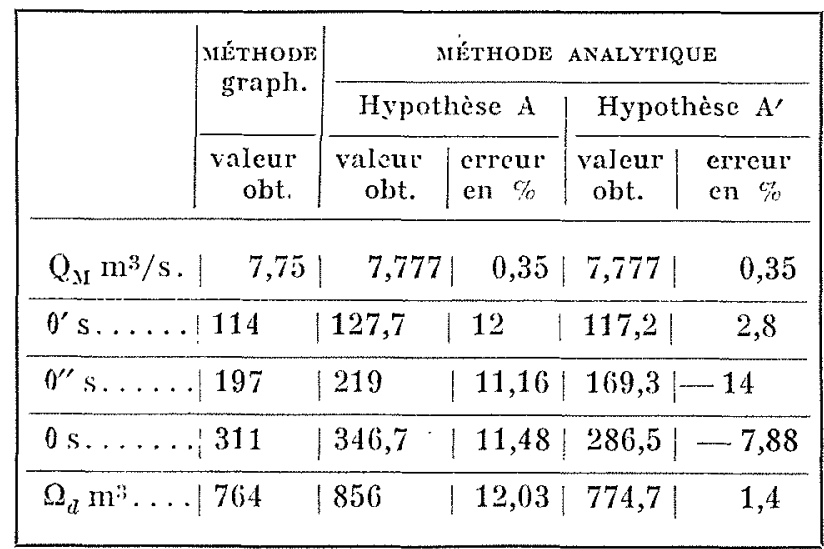

la méthode graphique classique, puis par la première méthode analytique approchée exposée dans cette étude (hypothèse A) et enfin en tenant compte de la substitution de $A^{\prime}$ à $A$ (hypothèse $\mathrm{A}^{\prime}$ ), les trois valeurs à comparer :

- du débit maximum $\mathrm{Q}_{\mathrm{M}}$;

- du volume total déversé $\Omega_{d}$;

- de la durée $\theta^{\prime}$ relative au déversement avec $\mathrm{W}>0$;

- de la durée $0^{\prime \prime}$ relative au déversement avec $\mathrm{W}<0$;

- de la durée $\theta=0^{\prime}+\theta^{\prime \prime}$ relative au déversement total,

ainsi que les erreurs relatives correspondant à la comparaison des résultats obtenus au moyen de l'une ou l'autre des méthodes analytiques approchées, aux valeurs fournies par la méthode graphique classique.

Pour les figures, convenons d'appeler, par exemple $\Omega_{d_{\mathrm{G}}}, \Omega_{d_{\mathrm{A}}}, \mathrm{Q}^{{ }^{\prime}{ }^{\prime}}$, les volumes $\Omega_{d}$ calculés par la méthode graphique $\left(\Omega_{a_{G}}\right)$, par la première méthode exposée dans la présente étude $\left(\Omega_{d_{A}}\right)$ et par la méthode indiquée en second lieu et substituant $A^{\prime}$ et $A\left(\Omega_{A}\right)$; adoptons une convention analogue pour $\theta_{i}, \theta_{n}, \theta_{n} \cdot$, ete.

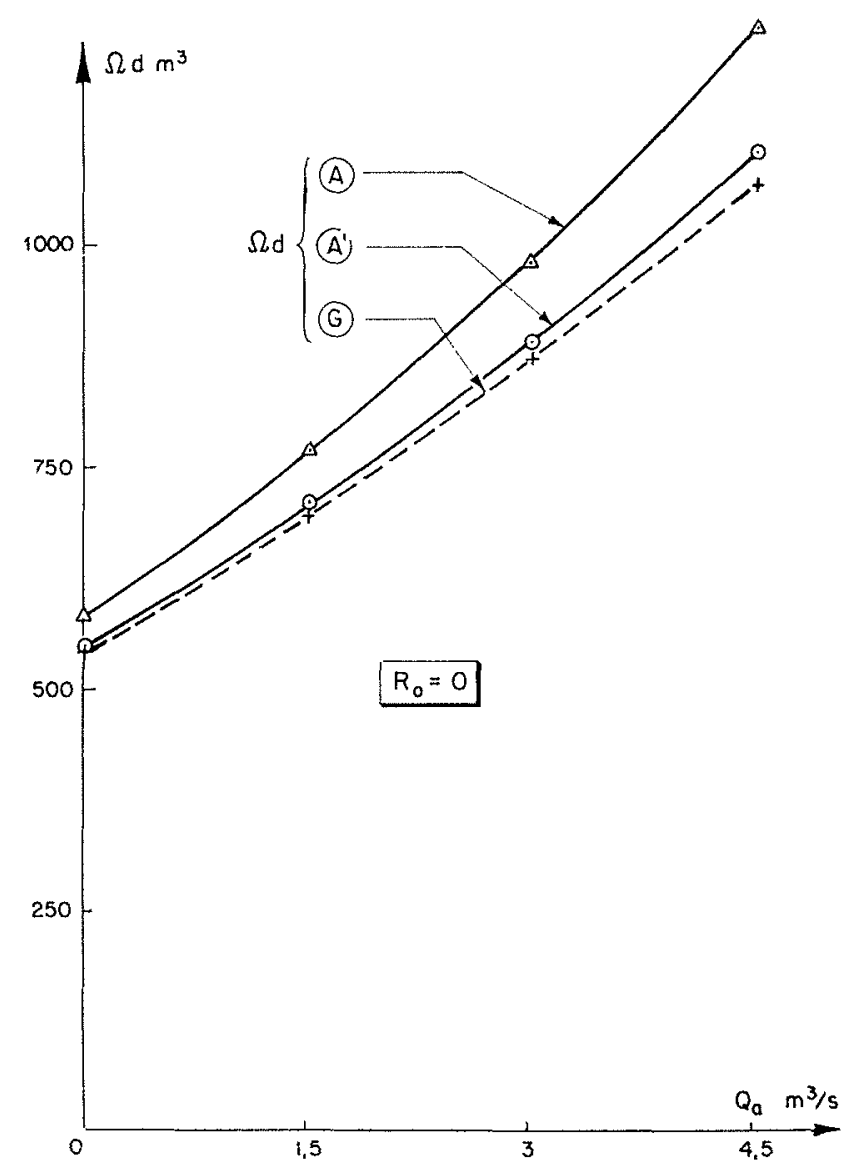

Fig. 2 


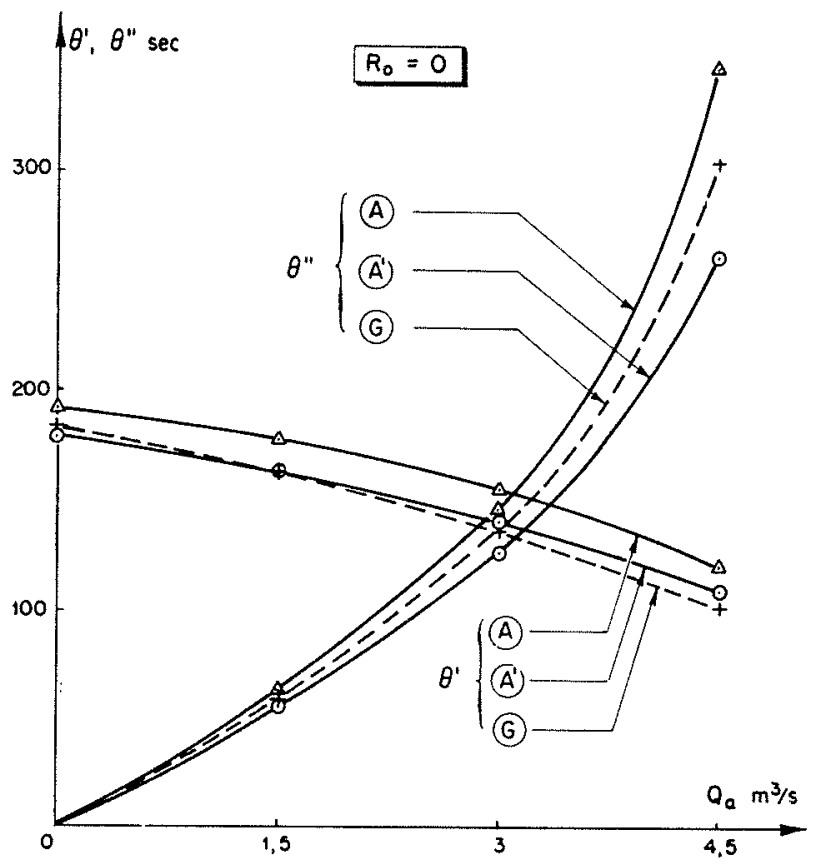

Fir. 3

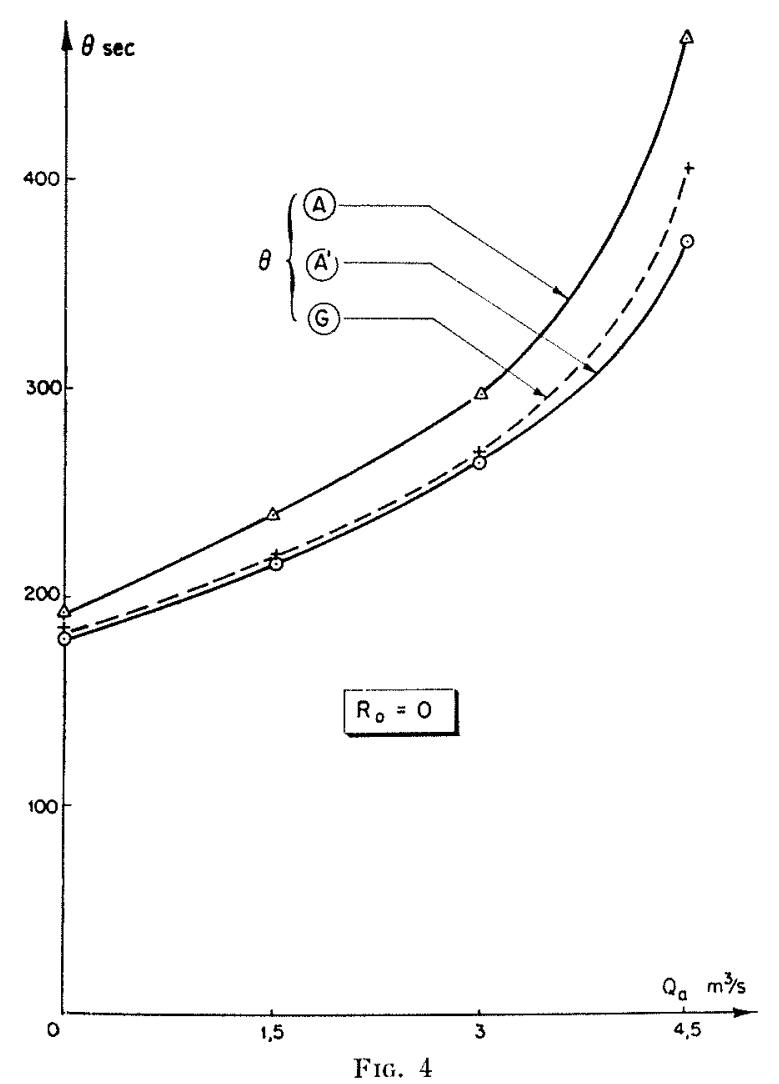

Nous avons représenté, en fonction de $Q_{a}$ :

- les courbes donnant les valeurs de $\Omega_{d_{G}}, \Omega_{d_{A}}$, $\Omega_{a_{A}}^{\prime}$, sur la figure 2 pour $R_{0}=0$, et sur la figure 6 pour $R_{0}=10 \mathrm{~m}$;

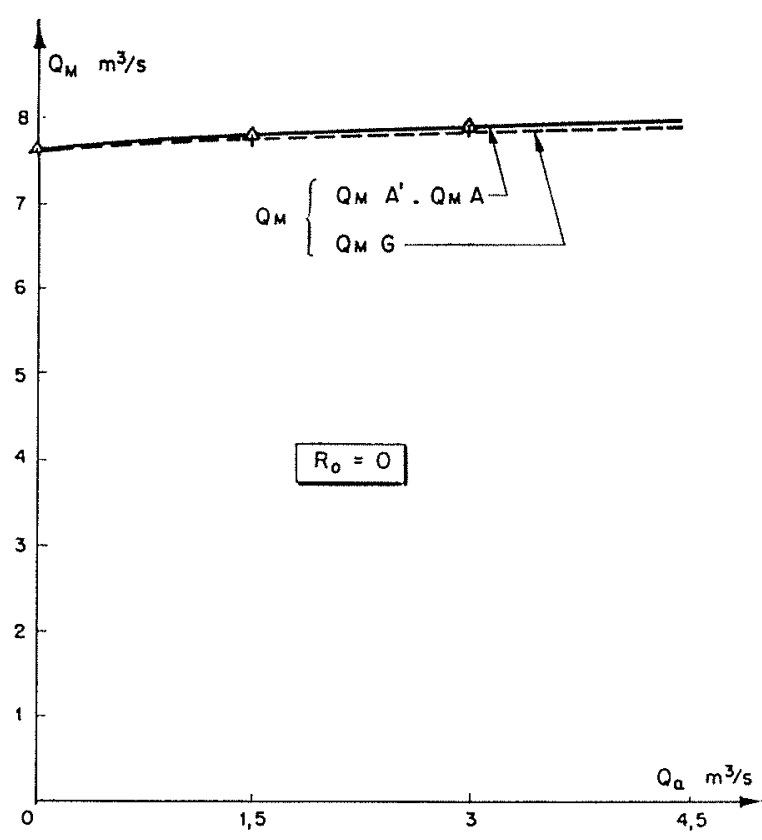

FIG. 5

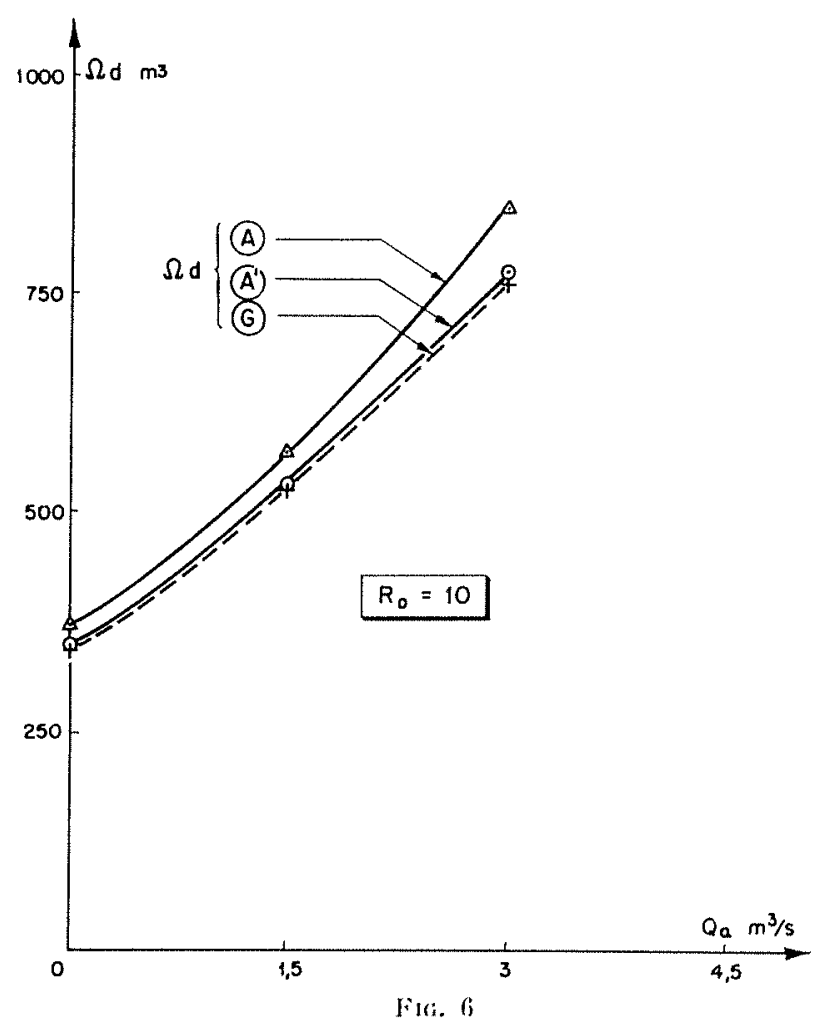

- les courbes donnant les valeurs de $\theta^{\prime}, \theta_{A}^{\prime}, \theta_{A^{\prime}}$. el de $\theta^{\prime \prime}{ }_{A}, 0^{\prime \prime}, 0^{\prime \prime}{ }^{\prime}$, sur la figure 3 pour $R_{0}=0$ et sur la figure 7 pour $R_{0}=10 \mathrm{~m}$; 


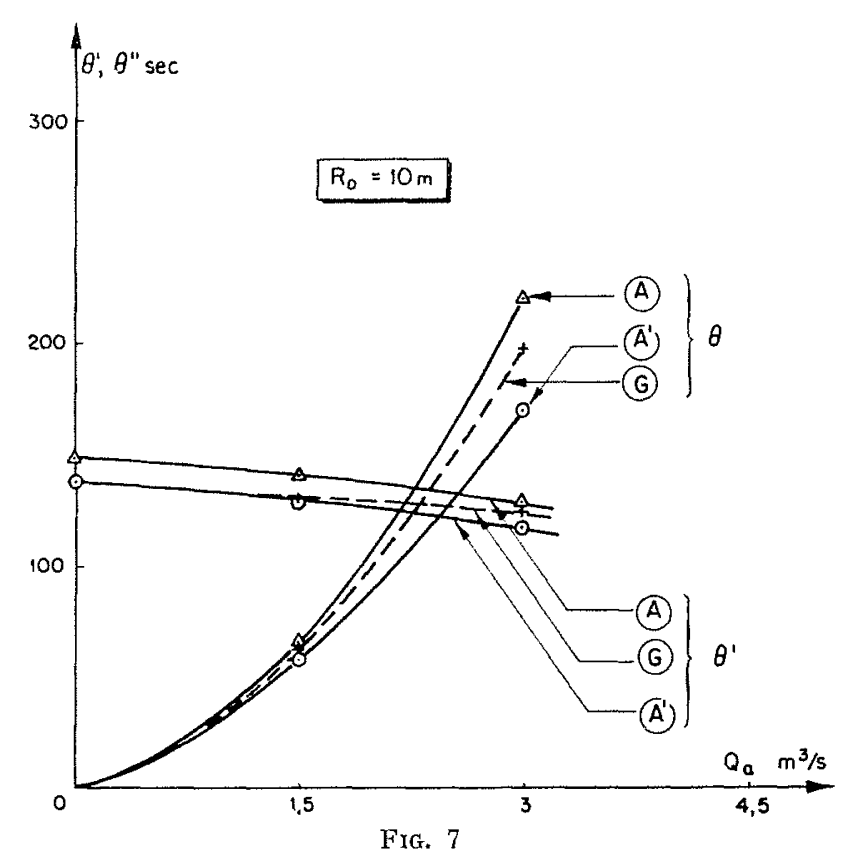

- les courbes donnant les valeurs de $0_{G}, \theta_{A}^{\prime}, 0^{\prime}{ }^{\prime}$ sur la figure 4 pour $R_{0}=0$ et sur la figure 8 pour $\mathrm{R}_{0}=10 \mathrm{~m}$;

- les courbes domnant les valeurs de $Q_{\mathrm{MrG}}, Q_{\mathrm{MA}}$, $Q_{\mathrm{MA}}$, sur la figure 5 pour $R_{0}=0$ et sur la figure 9 pour $R_{0}=10 \mathrm{~m}$.

\section{Remarque}

Les comparaisons précédentes permettent de se rendre compte du degré d'approximation que I'on obtient en négligeant, dans le calcul, les variations de la charge au-dessus du déversoir et en considérant une contre-pression moyenne constante.

Nous avons voulu nous rendre compte également des degrés de précision comparés du calcul analytique et de la méthode graphique, en nous plaçant dans le cas d'un seuil de longueur infinie pour lequel on a $h_{1}=0$ et pour lequel, par conséquent, le caractère approximatif de la méthode analytique disparaitt.

Dans ce but, nous avons, à partir des mêmes données numériques que ci-dessus, en nous limitant au cas de $R_{0}=0$ et en considérant successivement les quatre débits d'apport :

$$
\mathrm{Q}_{a}=\left\{\begin{array}{l}
0 \mathrm{~m}^{3} / \mathrm{s} \\
1,5 \mathrm{~m}^{3} / \mathrm{s} \\
3 \mathrm{~m}^{3} / \mathrm{s} \\
4,5 \mathrm{~m}^{3} / \mathrm{s}
\end{array} \quad \text { avec } \mathrm{A}=3 \mathrm{~m}\right.
$$

effectuć les constructions graphiques correspondantes.

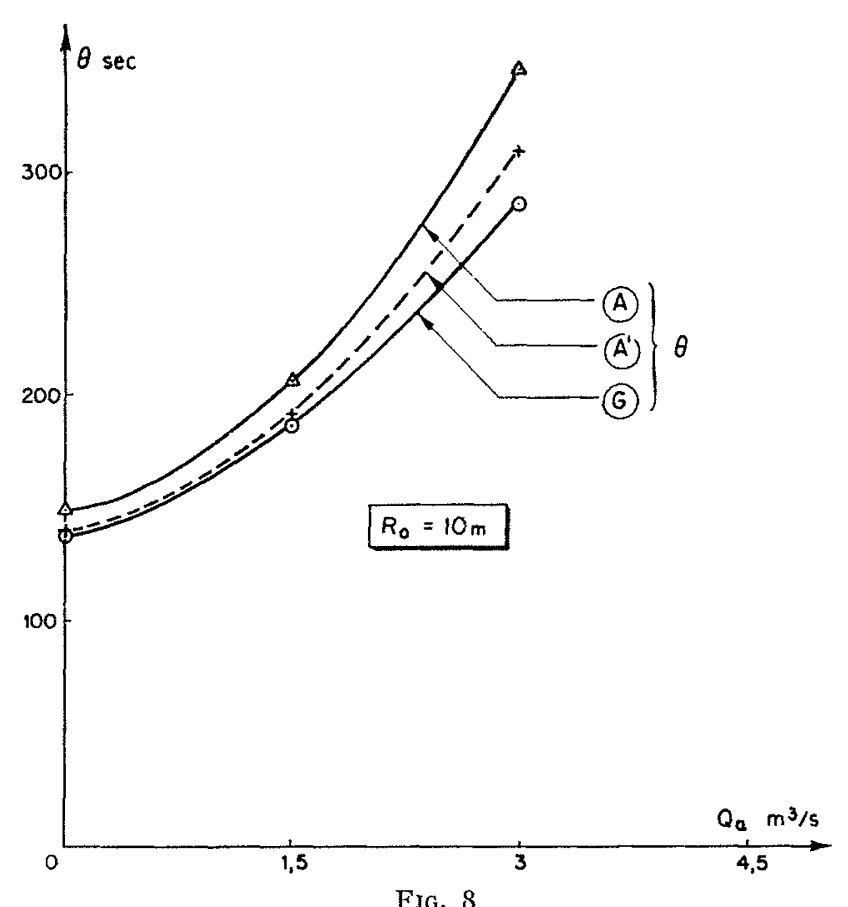

FIG. 8

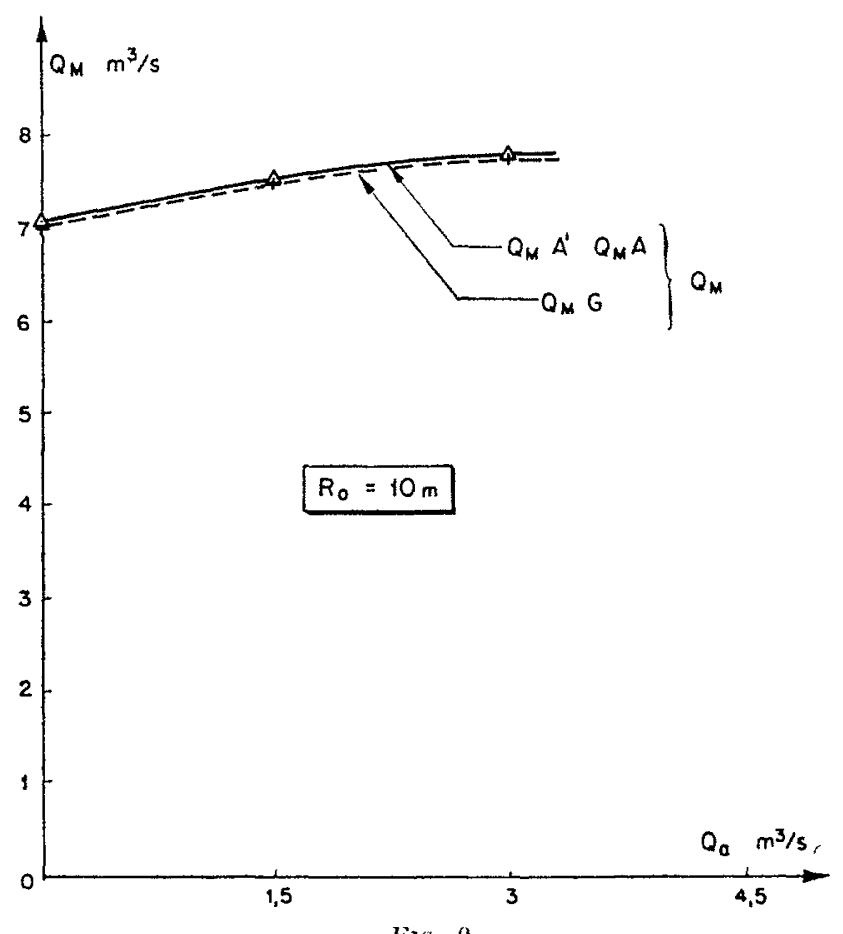

Fis. 9

Les tableaux VIII à XI donnent, en fonction de $\mathrm{Q}_{n}$, calculées respectivement par la méthode graphique, puis par la méthode analytique, les deux valeurs à comparer :

- du débit maximum $Q_{M}$,

- du volume total déversé $\Omega_{d}$,

- de la durće $\sigma^{\prime}$ du déversement avec $\mathrm{W}>0$, 
TABLEAU VIII

$\mathrm{Q}_{a}=0 \mathrm{~m}^{3} / \mathrm{s} \quad \mathrm{R}_{0}=0 \mathrm{~m}$

\begin{tabular}{|c|c|c|c|c|}
\hline & \multicolumn{3}{|c|}{ MÉTHoDe } & $\begin{array}{c}\text { Ecart } \\
\text { en } \%\end{array}$ \\
\cline { 2 - 5 } & graphique & analytique & \\
\hline$Q_{\mathrm{MI}} \mathrm{m}^{3} / \mathrm{s} \ldots \ldots$ & 7,65 & 7,61 & $-0,52$ \\
\hline$\theta^{\prime} \mathrm{s} \ldots \ldots \ldots$ & 194 & 191,6 & $-1,23$ \\
\hline$\theta^{\prime \prime} \mathrm{s} \ldots \ldots \ldots$ & & & & \\
\hline$\theta \mathrm{s} \ldots \ldots \ldots$ & 194 & 191,6 & $-1,23$ \\
\hline$\Omega_{a} \mathrm{~m}^{3} \ldots \ldots \ldots$ & 581,6 & 580 & $-0,27$ \\
\hline
\end{tabular}

TABLEAU IX

$$
\mathrm{Q}_{a}=1,5 \mathrm{~m}^{3} / \mathrm{s} \quad \mathrm{R}_{0}=0 \mathrm{~m}
$$

\begin{tabular}{|c|c|c|c|}
\hline & \multicolumn{2}{|c|}{ MÉTHODE } & \multirow{2}{*}{$\begin{array}{l}\text { Ecart } \\
\text { en } \%\end{array}$} \\
\hline & graphique & analytique & \\
\hline $\mathrm{Q}_{\mathrm{M}} \mathrm{m}^{3} / \mathrm{s} \ldots$ & 7,78 & 7,78 & 0 \\
\hline$\theta^{\prime} s \ldots \ldots \ldots$ & 175 & 177,8 & 1,6 \\
\hline$\theta^{\prime \prime} \mathrm{s} \ldots \ldots \ldots$ & 64,8 & 63,1 & $-2,6$ \\
\hline$\theta \mathrm{s} \ldots \ldots \ldots$ & 239,8 & 240,9 & 0,46 \\
\hline$\Omega_{d} \mathrm{~m}^{3} \ldots \ldots$ & 760 & 774,8 & 1,95 \\
\hline
\end{tabular}

TABLEAU $\mathrm{X}$

$$
\mathrm{Q}_{a}=3 \mathrm{~m}^{3} / \mathrm{s} \quad \mathrm{R}_{0}=0 \mathrm{~m}
$$

\begin{tabular}{|c|c|c|c|}
\hline & \multicolumn{2}{|c|}{ MLÉTHODE } & \multirow{2}{*}{$\begin{array}{l}\text { Ecart } \\
\text { en } \%\end{array}$} \\
\hline & graphique & analytique & \\
\hline $\mathrm{Q}_{\mathrm{M}} \mathrm{m}^{3} / \mathrm{s} \ldots \ldots$ & 7,85 & 7,88 & 0,38 \\
\hline$\theta^{\prime} s \ldots \ldots \ldots$ & 151.5 & 154 & 1,65 \\
\hline$\theta^{\prime \prime} \mathrm{s} \ldots \ldots \ldots$ & 143,5 & 143,8 & 0,21 \\
\hline$\theta \mathrm{s} \ldots \ldots \ldots$ & 295 & 297,8 & 0,95 \\
\hline$\Omega_{d} \mathrm{~m}^{3} \ldots \ldots \cdot$ & 977 & $\begin{array}{l}988,7 \\
\end{array}$ & 1,2 \\
\hline
\end{tabular}

TABLEAU XI

\begin{tabular}{|c|c|c|c|}
\hline & \multicolumn{2}{|c|}{ MÉTHODE } & \multirow{2}{*}{$\begin{array}{l}\text { Ecart } \\
\text { en } \%\end{array}$} \\
\hline & graphique & analytique & \\
\hline $\mathrm{Q}_{\mathrm{M}} \mathrm{m}^{3} / \mathrm{s} \ldots$ & 7,93 & 7,93 & 0 \\
\hline$\theta^{\prime} s \ldots \ldots \ldots$ & 119 & 120,3 & 1,09 \\
\hline$\theta^{\prime \prime}$ s........ & 340 & 349 & 2,65 \\
\hline$\theta s \ldots \ldots \ldots$ & 459 & 469,3 & 2,25 \\
\hline$\Omega_{d l} \mathrm{~m} ; \ldots \ldots$ & 1238 & 1255 & 1,37 \\
\hline
\end{tabular}

$$
\mathrm{Q}_{a}=4,5 \mathrm{~m}^{3} / \mathrm{s} \quad \mathrm{R}_{0}=0 \mathrm{~m}
$$

- de la durée $0^{\prime \prime}$ du déversement avec $W<0$, - de la durée $0=0^{\prime}+\sigma^{\prime \prime}$ du déversement total ainsi que les erreurs relatives correspondantes :

$$
\frac{\mathrm{Q}_{\mathrm{MA}}-\mathrm{Q}_{\mathrm{MF}}}{\mathrm{Q}_{\mathrm{MG}}} \quad, \quad \frac{\Omega_{t_{A}}-\Omega_{d_{G}}}{\Omega_{d_{\mathrm{G}}}} \text {, ete. }
$$

\section{Conclusion}

Comme on le voit, la méthode analytique basce sur l'hypothèse A conduit à des divergences appréciables. Au contraire, les écarts correspondant à l'emploi de la méthode analytique reposant sur l'hypothèse $\mathrm{A}^{\prime}$ demeurent toujours très faibles, et, de plus, en ce qui concerne l'élément essentiel, c'est-à-dire le volume déversé $\Omega_{l}$, ils sont dans le sens de la sécurité.

On peut donc conclure en faveur de la méthode analytique basce sur l'hypothìse $A^{\prime}$, d'une précision comparable à celle de la méthode graphique classique et d'un emploi plus simple et plus rapide.

Par ailleurs, les comparaisons effectuces entre la méthode analylique et la méthode graphique, dans le cas du seuil de longueur infinie pour lequel la méthode analytique devient rigoureuse, montrent que les résultals obtenus présentent le même caractère de précision.

\section{CAS OU L'APPORT DE DEBIT S'EFFECTUE AU-DESSOUS DE L'ETRANGLEMENT}

Dans le chapitre précédent, nous avons exposé une méthode analytique pour le calcul approché des chambres d'équilibre déversantes dans lesquelles un débit d'apport arrive à la partie su- périeure de l'ouvrage, ou, de façon plus précise, au-dessus de l'étranglement dans le cas où la chambre comporte un tel dispositif.

Nous exposons maintenant les résultats que 
nous avons obtenus, en collaboration avec M. Dialinas, dans l'étude du même problème lorsque le débit d'apport débouche dans la chambre, au-dessous de l'étranglement (fig. 10).

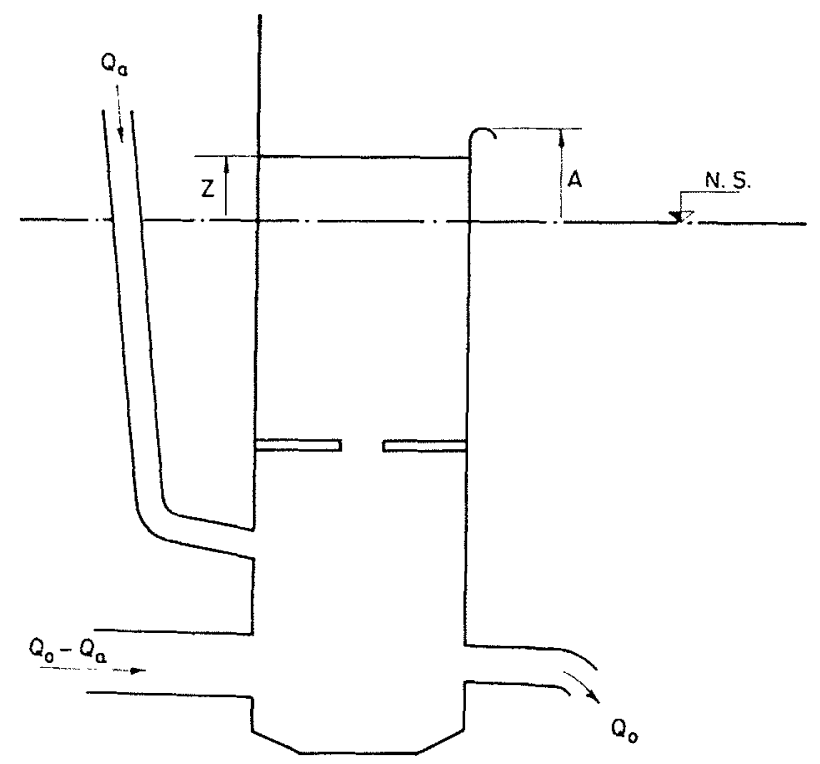

Fic. 10

\section{Phase préliminaire au déversement}

Les notations restant les mêmes qu'au chapitre I, les équations fondamentales des oscillations s'écrivent dans ce cas entre grandeurs relatives :

$$
\begin{aligned}
& v(d v / d z)+z+\varepsilon p+\varepsilon^{\prime} l=0 \\
& w=v-w_{a} \\
& p=p_{0} w^{2}=p_{0}\left(v--w_{a}\right)^{2} \\
& r=r_{0} v^{2}
\end{aligned}
$$

$\varepsilon$ et $\varepsilon^{\prime}$ ayant pour valeur absolue l'unité et pour signe celui de $w$ pour $\varepsilon$, celui de $v$ pour $\varepsilon^{\prime}$.

On remarque que, pendant toute la phase préliminaire au déversement, $\mathrm{W}$ et $\mathrm{V}$ restent positifs et l'on a :

$$
\varepsilon=\varepsilon^{\prime}=+1
$$

Les équations des oscillations ne sont pas intégrables analytiquement, mais la méthode graphique déjà rappelée (1) permet de déterminer la valeur $w w_{1}$ de $w$ à l'instant où débute le déversement.

On en déduit les valeurs $W_{1}=w_{1} W_{0}$ et $\mathrm{Q}_{1}=f \mathrm{~W}_{1}=w_{1} \mathrm{Q}_{0}$ de la vitesse $\mathrm{W}_{1}$ dans le canal d'amenće et du débit $Q_{1}$ débouchant de ce canal dans la chambre d'ćquilibre à l'instant où commence le déversement.

(1) Loc. cit., p. 2, note 1.

\section{Etude du déversement dans l'hypothèse d'un seuil de longueur infinie}

Dans l'hypothèse d'un seuil déversant de longucur infinie, les équations générales s'écrivent:

$$
\begin{gathered}
\frac{\mathrm{L}}{g} \frac{d \mathrm{~W}}{d t}+\mathrm{A}+\varepsilon \mathrm{P}+\varepsilon^{\prime} \mathrm{R}=0 \\
\mathrm{Q}_{d}=\mathrm{Q}_{c}+\mathrm{Q}_{u} \\
\mathrm{P}=\mathrm{P}_{0} \begin{array}{c}
\mathrm{Q}_{c}{ }^{2} \\
\mathrm{Q}_{0}{ }^{2}
\end{array} \quad \mathrm{R}=\mathrm{R}_{0} \frac{\left(\mathrm{Q}_{c}+\mathrm{Q}_{u}\right)^{2}}{\mathrm{Q}_{0}{ }^{2}}
\end{gathered}
$$

$Q_{d}$ et $Q_{c}$ désignant le débit déversant et le débit du canal d'amenée à l'instant $t$.

Au début, $\varepsilon$ et $\varepsilon^{\prime}$ sont positifs : $\varepsilon^{\prime}$ le restera jusqu'à la fin du déversement tandis que $\varepsilon$ deviendra négatif un peu avant la fin de celui-ci quand W changera de signe.

Ơn a donc l'équation :

$$
\frac{\mathrm{L}}{g f} \frac{d \mathrm{Q}_{c}}{d t}+\mathrm{A}+\mathrm{P}_{0} \frac{\mathrm{Q}_{c}{ }^{2}}{\mathrm{Q}_{0}{ }^{2}}+\mathrm{R}_{0} \frac{\left(\mathrm{Q}_{c}+\mathrm{Q}_{l}\right)^{2}}{\mathrm{Q}_{0}{ }^{2}}=0
$$

dont on tire :

$$
d t=-\frac{\mathrm{LQ}_{0}{ }^{2}}{g f} \frac{d \mathrm{Q}_{c}}{\left(\mathrm{P}_{0}+\mathrm{R}_{0}\right) \mathrm{Q}_{c}{ }^{2}+2 \mathrm{R}_{0} \mathrm{Q}_{a} \mathrm{Q}_{c}+\mathrm{AQ}_{0}{ }^{2}+\mathrm{R}_{0} \mathrm{Q}_{a}{ }^{2}}
$$

L'intégrale de cette fraction rationnelle donne, en prenant comme origine du temps le début du déversement :

$$
\begin{aligned}
t=\mathrm{T}^{\prime}[\operatorname{arctg} & \frac{\left(\mathrm{P}_{n}+\mathrm{R}_{0}\right) \mathrm{Q}_{1}+\mathrm{R}_{0} \mathrm{Q}_{a}}{\sqrt{\Delta^{\prime}}} \\
& \left.-\operatorname{arctg} \frac{\left(\mathrm{P}_{0}+\mathrm{R}_{0}\right) \mathrm{Q}_{c}+\mathrm{R}_{0} \mathrm{Q}_{n}}{\sqrt{\Delta^{\prime}}}\right]
\end{aligned}
$$

avec :

$$
\Delta^{\prime}=\left(\mathrm{P}_{0}+\mathrm{R}_{0}\right) \mathrm{AQ}_{0}{ }^{2}+\mathrm{P}_{0} \mathrm{R}_{0} \mathrm{Q}_{a^{\prime}}{ }^{2} \quad \text { et } \quad \mathrm{T}^{\prime}=\frac{\mathrm{LQ}_{0}{ }^{2}}{g f \sqrt{\Delta^{\prime}}}
$$

$-\Delta^{\prime}$ étant le discriminant toujours négatif du trinôme dénominateur de l'expression intégrće.

On en déduit :

$$
\begin{aligned}
\operatorname{arctg} & \frac{\left(\mathrm{P}_{0}+\mathrm{R}_{0}\right) \mathrm{Q}_{c}+\mathrm{R}_{0} \mathrm{Q}_{a}}{\sqrt{\Delta^{\prime}}} \\
& =\operatorname{arctg} \frac{\left(\mathrm{P}_{0}+\mathrm{R}_{0}\right) \mathrm{Q}_{1}+\mathrm{R}_{0} \mathrm{Q}_{a}}{\sqrt{\Delta^{\prime}}}-\frac{t}{\mathrm{~T}^{\prime}}
\end{aligned}
$$

et le débit déversant $\mathrm{Q}_{d}^{\prime}$ :

$$
\begin{array}{r}
Q_{d}^{\prime}=\frac{\sqrt{\Delta^{\prime}}}{\mathrm{P}_{0}+\mathrm{R}_{0}} \frac{\left(\mathrm{P}_{0}+\mathrm{R}_{0}\right) \mathrm{Q}_{1}+\mathrm{R}_{0} \mathrm{Q}_{a}-\sqrt{\Delta^{\prime}} \operatorname{tg}\left(t / \mathrm{T}^{\prime}\right)}{\sqrt{\Delta^{\prime}}+\left[\left(\mathrm{P}_{0}+\mathrm{R}_{0}\right) \mathrm{Q}_{1}+\mathrm{R}_{0} \mathrm{Q}_{\mathrm{a}}\right] \operatorname{tg}\left(t / \mathrm{T}^{\prime}\right)} \\
+\frac{\mathrm{P}_{0}}{\mathrm{P}_{0}+\mathrm{R}_{0}} \mathrm{Q}_{a} \quad(20)
\end{array}
$$


Ce débit est évidemment maximum et égal à :

$$
\mathrm{Q}_{\mathrm{M}}=\mathrm{Q}_{1}+\mathrm{Q}_{\mathrm{a}}
$$

à l'instant zéro du début du déversement, d'après l'hypothèse de base, relative au seuil.

Les relations (18), (19), (20) sont valables jusqu'à l'instant $\theta^{\prime}$ où $Q_{c}$ s'annule, instant donné, d'après (18) par :

$0^{\prime}=\mathrm{T}^{\prime}\left[\operatorname{arctg} \frac{\left(\mathrm{P}_{0}+\mathrm{R}_{0}\right) \mathrm{Q}_{1}+\mathrm{R}_{0} \mathrm{Q}_{a}}{\sqrt{\Delta^{\prime}}}-\operatorname{arctg} \frac{\mathrm{R}_{0} \mathrm{Q}_{a}}{\sqrt{\Delta^{\prime}}}\right]$

ou :

$$
0^{\prime}=\mathrm{T}^{\prime} \operatorname{arctg} \frac{\left(\mathrm{P}_{0}+\mathrm{R}_{0}\right) \mathrm{Q}_{1} \sqrt{\Delta^{\prime}}}{\Delta^{\prime}+\mu \cdot \mathrm{R}_{0} \mathrm{Q}_{a}}
$$

en posant :

$$
\mu=\left(\mathbf{P}_{0}+\mathbf{R}_{0}\right) \mathbf{Q}_{1}+\mathrm{R}_{0} \mathbf{Q}_{a}
$$

Le volume déversé, de 0 à $\theta^{\prime}$, est donné par :

$$
\Omega_{d}^{\prime}=\int_{0}^{\theta^{\prime}} \mathrm{Q}_{d}^{\prime} d t
$$

L'intégration, comple tenu de la valeur (20) de $Q_{d}^{\prime}$, s'effectue en posant :

$$
u=\operatorname{tg} t / \mathrm{T}^{\prime \prime}
$$

ce qui donne:

$$
d t=\mathrm{T}^{\prime} \frac{d u}{1+u^{2}}
$$

et, en appelant $u^{\prime}$ la valeur de $u$ à l'instant $o^{\prime}$ :

$$
u^{\prime}=\operatorname{tg} \theta^{\prime} / \mathrm{T}^{\prime}=\frac{\left(\mathrm{P}_{\mathrm{n}}+\mathrm{R}_{0}\right) \mathrm{Q}_{1} \sqrt{\Delta^{\prime}}}{\Delta^{\prime}+\mu \cdot \mathrm{R}_{0} \mathrm{Q}_{u}}
$$

et :

$$
\Omega_{d}^{\prime}=\frac{1}{\mathrm{R}_{0}+\mathrm{P}_{0}}\left\lceil\frac{\mathrm{LQ}_{0}{ }^{2}}{g f} \log \left(\frac{\sqrt{\Delta^{\prime}}+\mu \cdot l^{\prime}}{\sqrt{\Delta^{\prime}\left(1+u l^{\prime 2}\right)}}\right)+\mathrm{P}_{0} \mathrm{Q}_{a} 0^{\prime}\right\rceil
$$

$\mathrm{W}, \mathrm{Q}_{c}$ et $\varepsilon$ deviennent négatifs, après l'instant $\sigma^{\prime}$, et l'équation à intégrer s'écrit, pendant toute la durée du déversement:

$$
\frac{\mathrm{L}}{g f} \frac{d \mathrm{Q}_{.}}{d t}+\mathrm{A}-\mathrm{P}_{0} \frac{\mathrm{Q}_{r}{ }^{2}}{\mathrm{Q}_{0}{ }^{2}}+\mathrm{R}_{0} \frac{\left(\mathrm{Q}_{c}+\mathrm{Q}_{a}\right)^{2}}{\mathrm{Q}_{0}{ }^{2}}=0
$$

on en tire :

$$
d l=-\frac{\mathrm{LQ}_{0}{ }^{2}}{g f} \cdot \frac{d \mathrm{Q}_{t}}{\left(\mathrm{R}_{0}-\mathrm{P}_{0}\right) \mathrm{Q}_{c}{ }^{2}+2 \mathrm{R}_{0} \mathrm{Q}_{a} \mathrm{Q}_{c}+\mathrm{AQ}_{0}{ }^{2}+\mathrm{R}_{0} \mathrm{Q}_{a}{ }^{2}}
$$

Le second membre est une fraction rationnelle et le discriminant $\Delta^{\prime \prime}$ du trinôme constituant le dénominateur a pour valeur :

$$
\Delta^{\prime \prime}=\left(\mathrm{P}_{0}-\mathrm{R}_{0}\right) \mathrm{AQ}_{0}{ }^{2}+\mathrm{P}_{0} \mathrm{R}_{0} \mathrm{Q}^{2}{ }^{2}
$$

Trois solutions sont possibles selon que $\Delta^{\prime \prime}$ est positif, négatif ou nul.

$\operatorname{CaS} N^{\circ} 1: \Delta^{\prime \prime}>0:$

Ce cas, qui sera toujours réalisé, d'après (25), si $\mathrm{P}_{0}$ est supérieur on égal à $\mathrm{R}_{0}$, condition suffisante mais non nécessaire, correspond à :

$$
\left(\mathrm{P}_{0}-\mathrm{R}_{01}\right) A Q_{01}{ }^{2}+\mathrm{P}_{00} \mathrm{R}_{0} \mathrm{Q}_{a}{ }^{2}>0
$$

ou :

$$
\mathrm{Q}_{a}^{2}>\left(\frac{1}{\mathrm{P}_{0}}-\frac{1}{\mathrm{R}_{0}}\right) \mathrm{AQ}_{0}{ }^{2}
$$

Dans ce cas, l'intégration de (24) conduit, en prenant comme nouvelle origine du temps l'instant $0^{\prime}$, à l'expression :

$$
t=\frac{T^{\prime \prime}}{2} \log \left(\frac{\beta\left|\alpha+\left(\mathbf{R}_{0}-\mathbf{P}_{0}\right) Q_{n}\right|}{\alpha\left|\beta-\left(\mathbf{R}_{0}-\mathrm{P}_{0}\right) \mathbf{Q}_{i}\right|}\right)
$$

avec :

$\mathrm{T}^{\prime \prime}=\frac{\mathrm{LQ}_{u}{ }^{2}}{g f \sqrt{\left|\Delta^{\prime \prime}\right|}}, \quad \alpha=\sqrt{\Delta^{\prime \prime}}+\mathrm{R}_{0} \mathrm{Q}_{a}, \beta=\sqrt{\Delta^{\prime \prime}}-\mathrm{R}_{n} \mathrm{Q}_{u}$ on en déduil :

$$
\mathrm{Q}_{e}=-\left(\mathrm{AQ}_{0}{ }^{2}+\mathrm{R}_{0} \mathrm{Q}_{a}{ }^{2}\right) \frac{e^{2 t / \mathrm{I}^{\prime \prime}}-1}{\alpha e^{2 / \mathrm{I}^{\prime \prime \prime}+\beta}+\beta}
$$

et le débit déversant $Q^{\prime \prime}{ }^{\prime}$ :

$$
\mathrm{Q}^{\prime \prime}{ }_{a}=\mathrm{Q}_{a}+\mathrm{Q}_{c}=\mathrm{Q}_{a}-\left(\mathrm{AQ}_{0}{ }^{2}+\mathrm{R}_{0} \mathrm{Q}_{a}{ }^{2}\right) \frac{e^{2 l / T^{\prime \prime}}-1}{\alpha e^{2 l^{\prime} \mathrm{T}^{\prime \prime}+\beta}}
$$

$\mathrm{Q}^{\prime \prime}{ }_{d}$ ne peut que décroître, el il est d'ailleurs facile, en formant la dérivée de $Q^{\prime \prime}{ }$ p par rapport au lemps, de vérifier que celle-ci est négative.

La valeur initiale de $Q^{\prime \prime}{ }_{d}$ étant positive, te débit déversant ne peut s'annuler si, quand $t$ tend vers l'infini, la limite $\mathrm{Q}^{\prime \prime}{ }_{l l}$ de $\mathrm{Q}^{\prime \prime}{ }_{l}$ est positive, c'est-à-dire si l'on a :

$$
\mathrm{Q}^{\prime \prime}{ }^{\prime}=\mathrm{Q}_{a} \cdot-\frac{\mathrm{AQ}_{01}{ }^{2}+\mathrm{R}_{0} \mathrm{Q}_{a}{ }^{2}}{\alpha}>0
$$

on :

$$
\mathrm{Q}^{\prime \prime}{ }^{\prime l l}=\frac{\mathrm{P}_{11} \mathrm{Q}_{a}-\cdots-\sqrt{\Delta^{\prime \prime}}}{\mathrm{P}_{0}-\mathrm{R}_{0}}>0
$$

ou, en remplaçant $\Delta^{\prime \prime}$ par sa valeur :

$$
\mathrm{P}_{0}\left(\frac{\mathrm{Q}_{a}}{\mathrm{Q}_{0}}\right)^{2}>\mathrm{A}
$$

Celte condition peut être obtenue directemenl, en remarquant qu'en l'absence de déversement, dans le régime permanent final, le débit d'apport $\mathrm{Q}_{a}$ traverse le canal d'amenéc dans le sens négatif, en créant une perte de charge $\mathrm{P}_{0}\left(\mathrm{Q}_{a} / \mathrm{Q}_{0}\right)^{2}$ forcément inférieure à A puisque, sans cela, le 
plan d'eau, dans la chambre, serait plus haut que le seuil du déversoir et il y aurait déversement.

On peut également remarquer que le débit déversant limite et le débit limite correspondant $Q_{r l}$ dans le canal d'amenée :

$$
\mathrm{Q}_{c l}=--\left(\mathrm{Q}_{a}-\mathrm{Q}^{\prime \prime}{ }^{\prime}\right)
$$

vérifient la relation :

$$
\mathrm{P}_{0} \frac{\mathrm{Q}^{2} r l}{\mathrm{Q}_{0}{ }^{2}}=\mathrm{A}+\mathrm{R}_{0} \frac{\mathrm{Q}^{\prime \prime 2} d l}{\mathrm{Q}_{0}{ }^{2}}
$$

qui exprime l'égalité de deux expressions différentes de la pression sous l'étranglement, dans le régime permanent final.

Examinons maintenant l'hypothèse inverse, celle où le déversement cesse, au bout d'un temps $0^{\prime \prime}$, compté à partir de $0^{\prime}$ pris comme origine, et où l'on a, d'après ce qui précède :

$$
\mathrm{P}_{0}\left(\frac{\mathrm{Q}_{a}}{\mathrm{Q}_{0}}\right)^{2}<\mathrm{A}
$$

En annulant le second membre de (12), on obtient :

$$
0^{\prime \prime}=\frac{\mathrm{T}^{\prime \prime}}{2} \log \left(\frac{\mathrm{AQ}_{0}{ }^{2}+\mathrm{O}_{n} \sqrt{\Delta^{\prime \prime}}}{\mathrm{AQ}_{0}{ }^{2}-\mathrm{Q}_{a} \sqrt{\Delta^{\prime \prime}}}\right)
$$

Le volume déversé, pendant $0^{\prime \prime}$, a pour valeur :

$$
\Omega^{\prime \prime}{ }_{d}=\int_{0}^{\theta^{\prime \prime}} \mathrm{Q}^{\prime \prime}{ }_{d} d t
$$

En remplaçant $Q^{\prime \prime}{ }_{d}$ par son expression (29) et en faisant le changement de variable classique :

$$
e^{2 t / \mathrm{T}^{\prime}}=u \quad d t=\frac{\mathrm{T}^{\prime \prime}}{2} \frac{d u}{u}
$$

on obtient une fraction rationnelle dont l'intégration conduit à l'expression finale :

$$
\frac{1}{\left.\mathrm{P}_{0}-\mathrm{R}_{0}\right)}\left[\left(\sqrt{\Delta^{\prime \prime}}+\mathrm{P}_{0} \mathrm{Q}_{a}\right) 0^{\prime \prime}+\frac{\mathrm{L}_{0} \mathrm{Q}^{2}}{g l} \log \left(\frac{\sqrt{\Delta^{\prime \prime}}-\mathrm{P}_{0} \mathrm{Q}_{n}}{\sqrt{\Delta^{\prime \prime}}-\mathrm{R}_{0} \mathrm{Q}_{a}}\right)\right]
$$

CAS $N^{\circ} 2: \Delta^{\prime \prime}<0$ :

Remarquons, lout d'abord, que la condition $\Delta^{\prime \prime} \leqslant 0$ peut s'écrire :

$$
\left(\mathrm{P}_{0}-\mathrm{R}_{0}\right) \mathrm{A}_{0}{ }^{2}+\mathrm{P}_{0} \mathrm{R}_{0} \mathrm{Q}_{a}{ }^{2} \leqslant 0
$$

cc qui exige $\mathrm{P}_{0}<\mathrm{R}_{0}$ et peut encore se mettre sous la forme :

$$
\mathrm{P}_{0} \frac{\mathrm{Q}_{a}{ }^{2}}{\mathrm{Q}_{0}{ }^{2}} \leqslant \mathrm{~A}\left(1-\frac{\mathrm{P}_{\mathrm{n}}}{\mathrm{R}_{0}}\right)
$$

Cette condition entraîne a fortiori la suivante :

$$
\mathrm{P}_{0} \frac{\mathrm{Q}_{11^{2}}{ }^{2}}{\mathrm{Q}_{0}{ }^{2}}<\mathrm{A}
$$

déjà mise en évidence pour le non-déversement dans le régime permanent final.

Le cas de $\Delta^{\prime \prime}<0$, que nous étudions actuellement, correspond donc à :

$$
\mathrm{Q}_{a}^{2}<\left(\frac{1}{\mathrm{P}_{0}}-\frac{1}{\mathrm{R}_{0}}\right) \mathrm{AQ}_{0}^{2}
$$

L'intégration de (24) conduit alors, en prenant comme nouvelle origine du temps l'instant $\theta^{\prime}$, à l'expression :

$t=\mathrm{T}^{\prime \prime}\left[\operatorname{arctg} \frac{\mathrm{R}_{0} \mathrm{Q}_{n}}{\sqrt{-\Delta^{\prime \prime}}}-\operatorname{arctg} \frac{\mathrm{R}_{0} \mathrm{Q}_{n}+\left(\mathrm{R}_{0}-\mathrm{P}_{0}\right) \mathrm{Q}_{a}}{\sqrt{-\Delta^{\prime \prime}}}\right]$

On en déduit :

$$
\begin{array}{r}
\mathrm{Q}_{c}=\frac{\sqrt{-\Delta^{\prime \prime}}}{\mathrm{R}_{0}-\mathrm{P}_{0}} \cdot \frac{\mathrm{R}_{0} \mathrm{Q}_{a}-\sqrt{-\Delta^{\prime \prime}} \operatorname{tg}\left(t / \mathrm{T}^{\prime \prime}\right)}{\sqrt{-\Delta^{\prime \prime}+\mathrm{R}_{0} \mathrm{Q}_{a} \operatorname{tg}\left(t / \mathrm{T}^{\prime \prime}\right)}} \\
-\frac{\mathrm{R}_{0} \mathrm{Q}_{u}}{\mathrm{R}_{0} \mathrm{P}_{0}}
\end{array}
$$

et le débit déversant :

$$
\begin{array}{r}
\mathrm{Q}^{\prime \prime}{ }_{a}=\mathrm{Q}_{c}+\mathrm{Q}_{a}=\frac{\sqrt{-\Delta^{\prime \prime}}}{\mathrm{R}_{0}-\mathrm{R}_{0} \mathrm{Q}_{a}-\sqrt{-\Delta^{\prime \prime}} \operatorname{tg}\left(t / \mathrm{T}^{\prime \prime}\right)} \\
\sqrt{-\Delta^{\prime \prime}+\mathrm{R}_{0} \mathrm{Q}_{a} \operatorname{tg}\left(t / \mathrm{T}^{\prime \prime}\right)} \\
-\frac{\mathrm{P}_{0} \mathrm{Q}_{a}}{\mathrm{R}_{0}-\mathrm{P}_{0}}
\end{array}
$$

s'annule à l'instanl $0^{\prime \prime}$, compté à partir de $\theta^{\prime}$ :

$$
0^{\prime \prime}=\mathrm{T}^{\prime \prime} \operatorname{arctg}\left(\frac{\sqrt{-\Delta^{\prime \prime}} \mathrm{Q}_{a}}{\mathrm{AQ}_{0}{ }^{2}}\right)
$$

Le volume déversé $Q^{\prime \prime}{ }_{d}$, pendant la durée $0^{\prime \prime}$, est :

$$
\Omega^{\prime \prime}{ }_{d}=\int_{0}^{\theta^{\prime \prime}} \mathrm{Q}^{\prime \prime}{ }_{d} d t
$$

En faisant le changement de variable:

$$
\operatorname{tg}\left(\frac{t}{\mathbf{T}^{\prime \prime}}\right)=u
$$

on obtient une fraction rationnelle dont l'intégration fournit la valeur de $\Omega^{\prime \prime}{ }_{d}$ :

$$
\begin{aligned}
\Omega^{\prime \prime}= & \frac{1}{\mathrm{R}_{0}-\mathrm{P}_{0}} \cdot \\
& \frac{\mathrm{LQ}_{0}{ }^{2}}{g f}\left\{\log \left[\frac{\mathrm{AQ}_{0}{ }^{2}+\mathrm{R}_{0} \mathrm{Q}_{0}{ }^{2}}{\sqrt{\mathrm{A}^{2} \mathrm{Q}_{0}{ }^{4}-\Delta^{\prime \prime} \mathrm{Q}_{a}{ }^{2}}}\right]\right. \\
& \left.-\frac{\mathrm{P}_{0} \mathrm{Q}_{a}}{\sqrt{-\Delta^{\prime \prime}}} \operatorname{arctg}\left(\frac{\sqrt{-\Delta^{\prime \prime}} \cdot \mathrm{Q}_{a}}{\mathrm{AQ}_{0}{ }^{2}}\right)\right\}
\end{aligned}
$$

Cas $\mathrm{N}^{\circ} 3: \Delta^{\prime \prime}=0$ :

Ce cas constitue la limite entre les deux précédents et il correspond à : 


$$
\mathrm{Q}_{a}^{2}=\left(\frac{1}{\mathrm{P}_{0}}-\frac{1}{\mathrm{R}_{0}}\right) \mathrm{AQ}_{0}^{2}
$$

Comme nous l'avons déjà indiqué (relations 36 et 33), il n'y' a pas, dans ce cas, de déversement dans le régime permanent final.

Les formules correspondant à ce cas peuvent ĉtre obtenues à partir des précédentes, en passant à la limite pour $\Delta^{\prime \prime}=0$, comme nous nous en sommes assurés.

Les calculs sont, toutefois, assez laborieux et il est beaucoup plus simple de traiter directement le problème.

En intégrant l'équation (24) dans ce cas où le discriminant du trinôme dénominateur $\left(\Delta^{\prime \prime}\right)$ est nul, on obtient :

$$
\begin{gathered}
t=\frac{\mathrm{LQ}_{0}{ }^{2}}{g f}\left[\frac{1}{\mathrm{R}_{0} \mathrm{Q}_{a}+\left(\mathrm{R}_{0}-\mathrm{P}_{0}\right) \mathrm{Q}_{c}}-\frac{1}{\mathrm{R}_{0} \mathrm{Q}_{a}}\right] \\
\mathrm{Q}_{a}=-\frac{1}{\mathrm{R}_{0}-\mathrm{P}_{0}} \cdot \frac{\mathrm{R}_{0}{ }^{2} \mathrm{Q}_{a}{ }^{2} g f t}{\mathrm{LQ}_{0}{ }^{2}+\mathrm{R}_{0} \mathrm{Q}_{a} g f t} \\
\mathrm{Q}_{d}{ }^{\prime \prime}=\mathrm{Q}_{a}-\frac{1}{\mathrm{R}_{0}-\mathrm{P}_{0}} \cdot \frac{\mathrm{R}_{0}{ }^{2} \mathrm{Q}_{0}{ }^{2} g f t}{\mathrm{LQ}_{0}{ }^{2}+\mathrm{R}_{0} \mathrm{Q}_{a} g f t} \\
\theta^{\prime \prime}=\frac{\mathrm{LQ}_{0}{ }^{2}}{g f} \cdot \frac{\mathrm{R}_{0}-\mathrm{P}_{0}}{\mathrm{R}_{0} \mathrm{P}_{0}} \cdot \frac{1}{\mathrm{Q}_{a}} \\
\frac{\mathrm{I}_{a} \mathrm{Q}_{0}{ }^{2}}{g f}\left[\frac{1}{\mathrm{R}_{0}-\mathrm{P}_{0}} \log \left(\frac{\mathrm{R}_{0}}{\mathrm{P}_{0}}\right)-\frac{1}{\mathrm{R}_{0}}\right]
\end{gathered}
$$

\section{Extension des formules précédentes au cas d'un seuil déversant de longueur finie}

Nous avons montré au chapitre I qu'on pouvait, avec une excellente approximation, passer des formules concernant le seuil déversant de longueur infinie à la cote $A$ à celles qu'il convenait d'appliquer pour un seuil de même cote mais de longueur finie.

$h_{1}$ désignant la charge nécessaire pour l'écoulement, en régime permanent, au-dessus du déversoir, du débit maximum $Q_{3}$, nous avons vu qu'il suffit de remplacer, dans les formules générales établies pour le déversement au-dessus du seuil de longueur infinie, le terme $A$ par le terme $A^{\prime}$ défini comme suit :

$$
\mathrm{A}^{\prime}=\mathrm{A}+0,5 h_{1}
$$

$A^{\prime}$ représente sensiblement la valeur moyenne de la contre-pression due à la pesanteur pendant le déversement.

Il convient d'observer, en effet, que dans toutes ces formules générales, exception faite pour la condition (33), le terme A-n'intervient que pour caractériser la contre-pression due à la cote du plan d'eau dans la chambre.

Au contraire, dans la condition (33), A repré- sente effectivement la cote du seuil du déversoir, considérée comme position du plan d'eau à partir de laquelle commence le déversement: c'est pourquoi, pour cette condition et pour elle seule, A doit êlre conservé el ne doit pas être remplacé par $A^{\prime}$.

Pour la même raison, le calcul de $Q_{1}$, antérieur au début du déversement, ne change pas el fait loujours intervenir A.

\section{Conclusion de l'étude théorique}

Résumons les résultats obtenus pour l'étude du volume déversé à la suite d'une manœuvre de fermeture complète instantance intéressanl le débit maximum $Q_{0}$ des turbines.

En premier lieu, par la méthode graphique que nous avons indiquée (1), on détermine la valeur du débit $Q_{1}$ qui débouche dans la chambre d'équilibre à l'instant où le plan d'eau atteint la cote A du seuil du déversoir.

En second lieu, on étudie la phase du déversement.

\section{Si l'on a :}

$$
P_{0} \frac{Q_{11}{ }^{2}}{Q_{0}{ }^{2}}>A
$$

un déversement subsiste dans le régime permanent final, et le volume déversé est infini.

Si, au contraire, on a :

$$
P_{0} \frac{Q_{0}{ }^{2}}{Q_{0}^{2}}<A
$$

le déversement esl limité.

Dans cette dernière hypothese, trois cas sont à envisager selon les valeur's relatives du carré du débit d'apport $Q_{a}{ }^{2}$ et de l'expression $\mathrm{E}$ définic par :

$$
E=\left(\frac{1}{P_{0}}-\frac{1}{R_{0}}\right) A Q_{0}{ }^{2}
$$

auxquelles correspondent des expressions différentes de la durée tolale $0=0^{\prime}+0^{\prime \prime}$ du déversement et du volume lotal déversé $\Omega_{d}=\Omega^{\prime}{ }_{a}+\Omega^{\prime \prime}{ }_{d}$.

Remarquons que $0^{\prime}$ et $\Omega^{\prime}{ }_{d}$ demeurent les mêmes dans les trois cas et sont donnés par:

$$
\theta^{\prime}=T^{\prime} \operatorname{arctg} \frac{\left(P_{0}+R_{0}\right) Q_{1} \sqrt{\Delta^{\prime}}}{\lrcorner^{\prime}+\mu \cdot R_{0} Q_{a}}
$$

avec :

$$
\Delta^{\prime}=\left(\mathrm{P}_{0}+\mathrm{R}_{0}\right) A Q_{0}{ }^{2}+\mathrm{P}_{0} \mathrm{R}_{0} \mathrm{Q}_{2}{ }^{2}
$$

(1) Loc. cil., p. 2, notc 1 . 


$$
\begin{gathered}
\mathrm{T}^{\prime}=\frac{\mathrm{LQ}_{0}{ }^{2}}{g f \sqrt{\Delta^{\prime}}} \\
\mu=\left(\mathrm{P}_{0}+\mathrm{R}_{0}\right) \mathrm{Q}_{1}+\mathrm{R}_{0} \mathrm{Q}_{a} \\
\Omega_{d}^{\prime}=\frac{1}{\mathrm{R}_{0}+\mathrm{P}_{0}}\left|\frac{\mathrm{LQ}_{0}^{2}}{g f} \log \left(\frac{\sqrt{\Delta^{\prime}}+\mu u^{\prime}}{\sqrt{\Delta^{\prime}\left(1+u^{\prime 2}\right)}}\right)+\mathrm{P}_{0} \mathrm{Q}_{a} 0^{\prime}\right|
\end{gathered}
$$

arec :

$$
u^{\prime}=\frac{\left(\mathrm{P}_{0}+\mathrm{R}_{0}\right) \mathrm{Q}_{1} \sqrt{\Delta^{\prime}}}{\Delta^{\prime}+\mu \cdot \mathrm{R}_{0} \mathrm{Q}_{a}}
$$

$1^{\mathrm{er}} \mathrm{CAS}: \mathrm{Q}_{a}^{2}>\mathrm{E}:$

$$
\theta^{\prime \prime}=\frac{\mathrm{T}^{\prime \prime}}{2} \log \left(\frac{\mathrm{AQ}_{0}^{2}+\mathrm{Q}_{a} \sqrt{\Delta^{\prime \prime}}}{\mathrm{AQ}_{0}{ }^{2}-\mathrm{Q}_{a} \sqrt{\Delta^{\prime \prime}}}\right)
$$

avec :

$$
\begin{aligned}
& \Delta^{\prime \prime}=\left(\mathrm{P}_{0}-\mathrm{R}_{0}\right) \mathrm{AQ}_{0}{ }^{2}+\mathrm{P}_{0} \mathrm{R}_{0} \mathrm{Q}_{a}{ }^{2} \\
& \mathrm{~T}^{\prime \prime}=\frac{\mathrm{LQ}_{0}{ }^{2}}{g f \sqrt{\left|\Delta^{\prime \prime}\right|}} \\
& \Omega^{\prime \prime}{ }_{d}=\frac{1}{\mathrm{P}_{0}-\mathrm{R}_{0}} \\
& {\left[\left(\sqrt{\Delta^{\prime \prime}}+\mathrm{P}_{0} \mathrm{Q}_{a}\right) \theta^{\prime \prime}+\frac{\mathrm{LQ}_{0}{ }^{2}}{g f} \cdot \log \left(\frac{\sqrt{\Delta^{\prime \prime}}-\mathrm{P}_{0} \mathrm{Q}_{a}}{\sqrt{\Delta^{\prime \prime}}-\mathrm{R}_{0} \mathrm{Q}_{a}}\right)\right]}
\end{aligned}
$$

$2^{\circ} \mathrm{CAS}: \mathrm{Q}_{a}{ }^{2}<\mathrm{E}:$

$$
\begin{aligned}
0^{\prime \prime}= & \mathrm{T}^{\prime \prime} \operatorname{arctg}\left(\frac{\sqrt{-\Delta^{\prime \prime}} \mathrm{Q}_{a}}{\mathrm{AQ_{0 } { } ^ { 2 }}}\right) \\
\Omega^{\prime \prime}{ }_{a}=\frac{1}{\mathrm{R}_{0}-\mathrm{P}_{0}} \cdot & \frac{\mathrm{LQ}_{0}{ }^{2}}{g f}\left\{\log \left[\frac{\mathrm{AQ}_{0}{ }^{2}+\mathrm{R}_{0} \mathrm{Q}_{a}{ }^{2}}{\sqrt{\mathrm{A}^{2} \mathrm{Q}_{0}{ }^{4}-\Delta^{\prime \prime} \mathrm{Q}_{a}{ }^{2}}}\right]\right. \\
& \left.-\frac{\mathrm{P}_{0} \mathrm{Q}_{a}}{\sqrt{-\Delta^{\prime \prime}}} \operatorname{arctg}\left(\frac{\sqrt{-\Delta^{\prime \prime}} \mathrm{Q}_{a}}{\mathrm{AQ}_{0}{ }^{2}}\right)\right\}
\end{aligned}
$$

$3^{\circ}$ CAS : $\mathrm{Q}_{a}{ }^{2}=\mathrm{E}$ :

$$
\begin{gathered}
0^{\prime \prime}=\frac{\mathrm{LQ}_{0}{ }^{2}}{g f} \cdot \frac{\mathrm{R}_{0}-\mathrm{P}_{0}}{\mathrm{R}_{0} \mathrm{P}_{0}} \cdot \frac{1}{\mathrm{Q}_{a}} \\
\Omega^{\prime \prime}{ }_{d}=\frac{\mathrm{LQ}_{0}{ }^{2}}{g f}\left[\frac{1}{\mathrm{R}_{0}-\mathrm{P}_{0}} \log \left(\frac{\mathrm{R}_{0}}{\mathrm{P}_{0}}\right)-\frac{1}{\mathrm{R}_{0}}\right]
\end{gathered}
$$

\section{Comparaison avec la méthode graphique}

Nous nous sommes limite au cas du seuil de longueur infinie à la cote $\mathrm{A}$; dans ces conditions, la méthode analytique est rigoureuse et la comparaison avec la méthode graphique a seulement

\begin{tabular}{|c|c|c|c|}
\hline & \multicolumn{2}{|c|}{ MÉTHODE } & \multirow{2}{*}{$\begin{array}{l}\text { Ecart } \\
\text { cn \% }\end{array}$} \\
\hline & graphique & / analytique & \\
\hline$Q_{\mathrm{MI}} \mathrm{m}^{3} / \mathrm{s} \ldots$ & 5,72 & 5,82 & 1,8 \\
\hline$\theta^{\prime} s \ldots \ldots \ldots$ & 112 & 110 & $-1,8$ \\
\hline$\theta^{\prime \prime} s \ldots \ldots$ & 61 & 60,8 & $-0,33$ \\
\hline$\theta \mathrm{s} \ldots \ldots \ldots$ & 173 & 170,8 & $-0,7$ \\
\hline$\Omega_{d} \mathrm{~m}^{3} \ldots \ldots$ & 447,5 & | 451,15 & 0,82 \\
\hline
\end{tabular}
pour but de mettre en évidence les degrés de précision comparés des deux procédés.

Nous avons considéré le cas d'une usine cor-

\begin{tabular}{|c|c|c|}
\hline & MÉTHODE & \multirow{2}{*}{$\begin{array}{l}\text { Ecart } \\
\text { en } \%\end{array}$} \\
\hline & graphique | analytique & \\
\hline $\mathrm{Q}_{\mathrm{M}} \mathrm{m}^{3} / \mathrm{s} \ldots \ldots$ & $4,528 \mid$ & $-0,44$ \\
\hline$\theta^{\prime} s \ldots \ldots \ldots$ & 73,40 & $-0,81$ \\
\hline$\theta^{\prime \prime} \mathrm{s} \ldots \ldots \ldots$ & \begin{tabular}{l|l|}
126,86 \\
\end{tabular} & $-0,5$ \\
\hline $0 \mathrm{~s} \ldots \ldots \ldots$ & 200,26 & $-0,62$ \\
\hline$\Omega_{d} \mathrm{~m}^{3} \ldots \ldots$ & $\mid 515$ & -2 \\
\hline
\end{tabular}
respondant aux données suivantes :

$$
\begin{array}{rlrl}
\mathrm{L} & =4.138 \mathrm{~m} & \mathrm{P}_{0} & =8,5 \mathrm{~m} \\
\mathrm{I} & =3,464 \mathrm{~m}^{2} & \mathrm{~F} & =5,55 \mathrm{~m}^{2} \\
\mathrm{Q}_{0} & =8 \mathrm{~m}^{3} / \mathrm{s} & \mathrm{R}_{0} & =10 \mathrm{~m}
\end{array}
$$

$$
\mathrm{A}=3 \mathrm{~m}
$$

Les tableaux XII à XVI donnent en fonction de $Q_{a}$, calculées respectivement par la méthode

\section{TABLEAU XII}

$$
\mathrm{Q}_{a}=0 \mathrm{~m}^{3} / \mathrm{s} \quad \mathrm{R}_{0}=10 \mathrm{~m}
$$

\begin{tabular}{|c|c|c|c|}
\hline & \multicolumn{2}{|c|}{ MÉTHODE } & \multirow{2}{*}{$\begin{array}{l}\text { Ecart } \\
\text { en } \%\end{array}$} \\
\cline { 2 - 5 } & graphique & analytique & \\
\hline$Q_{\mathrm{MI}} \mathrm{m}^{3} / \mathrm{s} \ldots \ldots$ & 7,04 & 7,02 & 0,3 \\
\hline$\theta^{\prime} \mathrm{s} \ldots \ldots \ldots$ & 150 & 149,3 & $-0,47$ \\
\hline$\theta^{\prime \prime} \mathrm{s} \ldots \ldots \ldots$ & & & \\
\hline$\theta \mathrm{s} \ldots \ldots \ldots$ & 150 & 149,3 & $-0,47$ \\
\hline$\Omega_{d} \mathrm{~m}^{3} \ldots \ldots \ldots$ & 375 & 370 & $-1,34$ \\
\hline
\end{tabular}

\section{TABLEAU XIII}

$$
\mathrm{Q}_{a}=1,5 \mathrm{~m}^{3} / \mathrm{s} \quad \mathrm{R}_{0}=10 \mathrm{~m}
$$

\section{TABLEAU XIV}

$$
\mathrm{Q}_{a}=3 \mathrm{~m}^{3} / \mathrm{s} \quad \mathrm{R}_{0}=10 \mathrm{~m}
$$




\section{TABLEAU XV}

$\mathrm{Q}_{a}=4 \mathrm{~m}^{3} / \mathrm{s} \quad \mathrm{R}_{0}=10 \mathrm{~m}$

\begin{tabular}{|c|c|c|c|}
\hline & \multicolumn{2}{|c|}{ мЕ́тноDE } & \multirow{2}{*}{$\begin{array}{l}\text { Ecart } \\
\text { en } \%\end{array}$} \\
\hline & graphique & analytique & \\
\hline$Q_{M} m^{3} / s \ldots \ldots$ & 3,65 & 3,616 & $-0,83$ \\
\hline$\sigma^{\prime} s \ldots \ldots \ldots$ & 53 & 52,46 & -1 \\
\hline$\theta^{\prime \prime} \mathrm{s} \ldots \ldots \ldots$ & 199 & \begin{tabular}{l|l}
198,29
\end{tabular} & $-0,36$ \\
\hline $0 \mathrm{~s} \ldots \ldots \ldots$ & 252 & $\mid \quad 250,75$ & $-0,5$ \\
\hline$\Omega_{d} \mathrm{~m}^{3} \ldots \ldots$ & 579 & | 571,07 & $-1,38$ \\
\hline
\end{tabular}

\section{TABLEAU XVI}

$$
\mathrm{Q}_{a}=4,5 \mathrm{~m}^{3} / \mathrm{s} \quad \mathrm{R}_{0}=10 \mathrm{~m}
$$

\begin{tabular}{|c|c|c|c|c|}
\hline & \multicolumn{3}{|c|}{ MÉThode } & \multirow{2}{*}{$\begin{array}{c}\text { Ecart } \\
\text { en } \%\end{array}$} \\
\cline { 2 - 6 } & graphique | analytique & \\
\hline$Q_{\mathrm{M}} \mathrm{m}^{3} / \mathrm{s} \ldots \ldots$ & 3,16 & 3,18 & 0,6 \\
\hline$\theta^{\prime} \mathrm{s} \ldots \ldots \ldots$ & 44 & 43,5 & -1 \\
\hline$\theta^{\prime \prime} \mathrm{s} \ldots \ldots \ldots$ & 274 & $\mid$ & 290,1 & 5,9 \\
\hline $0 \mathrm{~s} \ldots \ldots \ldots$ & 318 & $\mid$ & 333,6 & 5 \\
\hline$\Omega_{d} \mathrm{~m}^{3} \ldots \ldots \ldots$ & 606,3 & 589,5 & $-2,8$ \\
\hline
\end{tabular}

graphique, puis par la méthode analytique, les deux valeurs à comparer :
- du débit maximum $Q_{\mathrm{N}}$,

- du volume total déversé $\Omega_{d}$,

- de la durée $\theta^{\prime}$ du déversement avec $W>0$,

- de la durée $\theta^{\prime \prime}$ du déversement avec $W<0$,

- de la durée $0=0^{\prime}+0^{\prime \prime}$ du déversement total ainsi que les erreurs relatives correspondantes :

$$
\frac{Q_{\mathrm{MA}}-Q_{\mathrm{Mr} C}}{Q_{\mathrm{MG}}} \quad, \quad \frac{\Omega_{d_{A}}-Q_{d_{G}}}{\Omega_{t_{\mathrm{G}}}} \text {, etc. }
$$

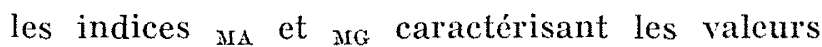
obtenues respectivement par le calcul analytique d'une part, et par la méthode graphique d'autre part.

On voit par l'examen de ces tableaux que la concordance est toujours très grande : c'est seulement dans le cas de $\mathrm{Q}_{a}=4,5 \mathrm{~m}^{3} / \mathrm{s}$ que l'écart $\operatorname{sur} \theta$ atteint $5 \%$ en raison des conditions particulières qui diminuent à la fois la précision du graphique et du calcul numérique, ce dernier faisant intervenir alors des différences de nombres très voisins l'un de l'autre.

\section{Conclusion}

La méthode proposée conduit donc à des résultats d'une précision tout à fait comparable à ceux de la méthode graphique, et son emploi est nolablement plus rapide que celui de celte dernière.

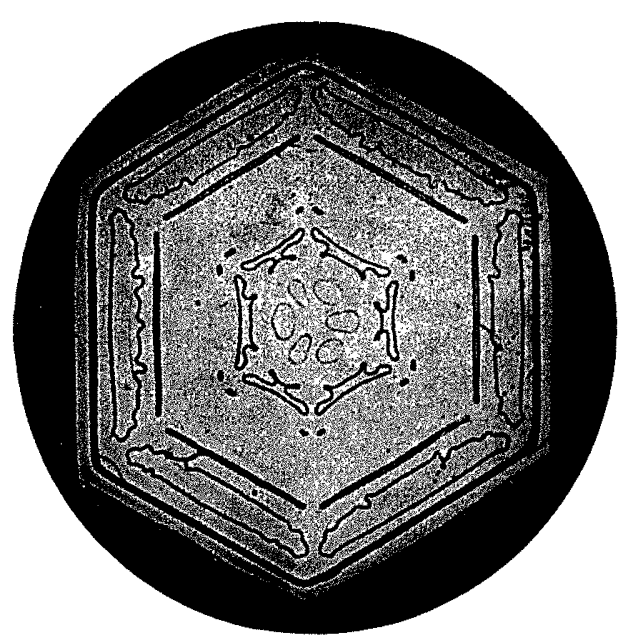

